\title{
Flume experiments on wind induced flow in static water bodies in the presence of protruding vegetation
}

\author{
Tirtha Banerjee $^{1}$, Marian Muste ${ }^{\mathrm{b}}$, Gabriel Katul ${ }^{\mathrm{c}}$ \\ ${ }^{a}$ Nicholas School of the Environment, Box 90328, Duke University, Durham, NC 27708, USA. \\ ${ }^{b}$ Dept. of Civil and Environmental Engineering, IIHR Hydroscience and Engineering, The Univ. of Iowa, Iowa City, IA \\ 52242. \\ ${ }^{c}$ Nicholas School of the Environment, Box 90328, Duke University, Durham, NC 27708, USA.
}

\begin{abstract}
The problem of wind-induced flow in inland waters is drawing significant research attention given its relevance to a plethora of applications in wetlands including treatment designs, pollution reduction, and biogeochemical cycling. The present work addresses the role of wind induced turbulence and waves within an otherwise static water body in the presence of rigid and flexible emergent vegetation through flume experimentation and time series analysis. Because no prior example of Particle Imaging Velocimetry (PIV) experiments involving air-water and flexible oscillating components have been found in the literature, a spectral analysis framework is needed and proposed here to guide the analysis involving noise, wave and turbulence separation. The experiments reveal that wave and turbulence effects are simultaneously produced at the airwater interface and the nature of their coexistence is found to vary with different flow parameters including water level, mean wind speed, vegetation density and its flexibility. For deep water levels, signature of fine-scaled inertial turbulence is found at deeper layers of the water system. The wave action appears stronger close to the air-water interface and damped by the turbulence deeper inside the water system. As expected, wave action is found to be dominated in a certain frequency range driven by the wind forcing, while it is also diffused to lower frequencies by means of (wind-induced) oscillations in vegetation. Regarding the mean water velocity, existence of a counter-current flow and its switching to fully forward flow in the direction of the wind under certain combinations of flow parameters were studied. The relative importance of wave and turbulence to the overall energy, degree of anisotropy in the turbulent energy components, and turbulent momentum transport at different depths from the air-water interface and flow combinations were then quantified. The flume experiments reported here differ from previous laboratory studies in the related literature involving vegetation in the sense that the wave forcing is only present on the water surface contrary to a full-body excitation by tidal wave simulators and thus important in advancing the knowledge regarding a wider range of water resource problems.
\end{abstract}

Keywords: Aquatic vegetation canopy, Canopy turbulence, Counter-current flow, Flexible vegetation, Flume experiments, Lorenz curve, PIV, Spectral analysis, Turbulent intensity, Vegetation drag, Wave intensity, Wave turbulence interaction, Wind induced flow.

Email addresses: tirtha.b@duke.edu (Tirtha Banerjee), marian-muste@uiowa.edu (Marian Muste), gaby@duke.edu (Gabriel Katul)

Preprint submitted to Advances in Water Resources

November 29, 2014

(C) 2014. This manuscript version is made available under the Elsevier user license http://www.elsevier.com/open-access/userlicense/1.0/ 


\section{Introduction}

The monetary value and multiple ecosystem services provided by static water bodies such as wetlands and marshes are rarely disputed (Begon and Harper, 1986; Bennett et al., 2002; Costanza et al., 1998; Fonseca and Fisher, 1986; Fonseca and Cahalan, 1992; Gambi et al., 1990; Gleason et al., 1979; Jordanova and James, 2003; Nepf, 1999, 2012a; Pergent-Martini et al., 2006; Prigent et al., 2001; Reusch and Chapman, 1995; Ward et al., 1984); however, characterization of the flow field, needed in all such ecosystem valuation, remains the subject of active research. Operational models for water flow in wetlands commonly assume the flow to be analogous to a wide and shallow open channel described by the so-called Saint-Venant equations that are then mathematically closed for the energy losses using a Manning-type formula with an associated friction factor as recently reviewed elsewhere (Katul et al., 2011). Because the flow depth in wetlands is shallow, wind effects can be sufficiently large so as to induce flow even in the absence of any bed slope. These wind effects on the flow have traditionally been lumped into changes in the friction factor, with little theoretical or experimental underpinning, which is the main motivation for this work. By no means this is a unique criticism to such an operational framework. Another common criticism is the lack of explicit inclusion of the effects of vegetation on both - bulk and turbulent flow quantities needed for the purposes here. Such vegetation characterization on the bulk flow has often been directed to drag or flow resistance estimation for unidirectional flow but in the absence of wind (Fairbanks and Diplas, 1998; Kubrak et al., 2008; Lee et al., 2004; Montakhab et al., 2012; Nepf, 1999; Nepf and Koch, 1999; Nepf and Vivoni, 2000; Nepf, 2012a; Righetti, 2008; Sukhodolov and Sukhodolova, 2009; Sun and Shiono, 2009; Tanino and Nepf, 2008; Wilson et al., 2003). A number of studies have also been concerned with detailed description of turbulent processes needed in modeling movement of particulate matter inside aquatic vegetation, characterization of dispersion, and lateral diffusion (Huang et al., 2008; Lightbody and Nepf, 2006; Murphy et al., 2007; Nepf et al., 1997a,b; Serra et al., 2004). In unidirectional flow through a vegetation canopy, the shear layer on top of a canopy generates canopy scale turbulence that is pushed down to the canopy displacement length (Nepf, 2012a; Poggi et al., 2004). At the bottom of the canopy, turbulence is generated by stem scale wakes (Nepf, 2012a; Poggi et al., 2004). In dense canopies, the intensity of turbulence is reduced by sheltering (Raupach and Thom, 1981; Finnigan, 2000), which plays a positive role in sediment retention and prevention of bed erosion (Baptist, 2003; Jordanova and James, 2003; Jordanova et al., 2006; Liu and Shen, 2008; Neumeier and Ciavola, 2004; Schmid et al., 2005; Sharpe and James, 2006; Shucksmith et al., 2011; Stephan et al., 2005; Zong and Nepf, 2011). However, all these experiments did not consider the problem of wind-induced flow within emergent aquatic vegetation, the main compass of this work.

Any wetland or channel featuring aquatic vegetation is naturally subjected to wind flow and wind-generated waves that can influence the flow-field inside the water body, which is further complicated by the presence of emergent vegetation also subjected to the wind and consequent oscillation depending upon their flexibility. In the absence of vegetation, the problem of wind blowing over a water surface is not particularly new and has a long history (Charnock, 1955; Csanady, 2004). Wind flowing over a static water body such as a lake or reservoir (as in the original work of Charnock) is the main source of mechanical energy for turbulent mixing inside the water body. The wind flowing over the water surface causes a drift current in the direction it blows thus perturbing the water surface, which is called wind set-up (Hellström, 1941; Tsanis, 1989). This local pressure gradient generated due to the 'tilt of the surface' (Tsanis, 1989) creates a reversed flow at the bottom of the water body, as well as ensuring mass continuity 
in a vertical plane. Here, the difference of the physical process governing the propagation of gravity waves and the wind set-up should be noted. Gravity wave is a self sustained process initiated out of an initial perturbation before the wave is damped. Whereas the wind set-up is continuously sustained by air motion, which injects energy into the water and applies shear stress on the surface. Studies like Baines and Knapp (1965); Cheung and Street (1988); Goossens et al. (1982); Komori et al. (1993); Kranenburg (1987); Langmuir et al. (1938); Longo et al. (2012); Sanjou et al. (2010); Sanjou and Nezu (2011); Tsanis and Leutheusser (1988); Tsanis (1989); Wu (1975) have reported experimental investigation of wind induced water currents, focusing on both the surface motion and the counter-current flow but without vegetation. Some studies have discussed a simple analytical model of wind set-up by constructing one, two and three dimensional models and engineering models for wind induced counter-current flow but without any vegetation (Bukatov and Zavyalov, 2004; Belcher et al., 1994; Cioffi et al., 2005; Harris et al., 1996; Hunter and Hearn, 1987; Jin and Kranenburg, 1993; Koçyigit and Koçyigit, 2004; Meyer, 2011; Rodi, 1980; Wu and Tsanis, 1995; Yang, 2001; Yang et al., 2008). The role of waves in vegetated system has been considered, but in these cases, the entire wave was imposed on the vegetation primarily to mimic tidal systems so as to study wave attenuation, equivalent bed roughness and friction factor inside aquatic vegetation canopy under wave forcing (Bradley and Houser, 2009; Fonseca and Cahalan, 1992; Hansen and Reidenbach, 2011; Infantes et al., 2012; Kobayashi et al., 1993; Manca et al., 2012; Mendez and Losada, 2004; Riffe et al., 2011; Thomas and Cornelisen, 2003). Others have also discussed the nature of the flow field inside a flexible aquatic vegetation under the action of wave forcing by means of laboratory experiments (Coops and Van der Velde, 1996; Gambi et al., 1990; Luhar et al., 2010; Lowe et al., 2005a; Reidenbach et al., 2007; Tanino et al., 2005; Tanino and Nepf, 2008) and by modeling (Li and Yan, 2007; Li and Zhang, 2010; Mullarney and Henderson, 2010). Flapping motion of the vegetation, a generic feature of many aquatic vegetation under oscillatory forcing like waves, also appears to enhance nutrient uptake (Koch and Gust, 1999; Huang et al., 2011; Lowe et al., 2005b; Nepf, 2012b; Thomas and Cornelisen, 2003). On similar lines, a few studies have addressed the characterization of turbulent structures and detection of sweep-ejection cycles and traveling vortex induced synchronous progressive waving action on aquatic flexible vegetation called 'Monami' (Ghisalberti and Nepf, 2002, 2006, 2009; Nepf and Ghisalberti, 2008; Okamoto and Nezu, 2013; Oldham and Sturman, 2001).

It is evident from this literature survey that progress has been made in understanding (i) the dynamics of wind-shear-water interaction without vegetation, and (ii) the flow dynamics in presence of flexible vegetation under wave forcing. Yet, all these previous studies in the second category have dealt with wave forcing generated by wave-makers, i.e., the whole water mass has been subjected to a wave forcing. Under this condition, some studies like Luhar et al. (2010), Lowe et al. (2005a) and Lowe et al. (2005b) have used linear wave theory to interpret their results - for example the decomposition of the instantaneous flow-field into phase averaged, coherent and turbulent components. Other studies examining counter-current flow without vegetation like Tsanis and Leutheusser (1988) and Tsanis (1989) have analyzed their results without any regard to linear wave theory and employed parabolic mixing length models to close their turbulent stresses.

The present work related to wind induced flow in a water body falls in the middle of these two aforementioned approaches. The presence of oscillating flexible vegetation increases the complexity of the problem. No previous reference of this problem has been found in the literature where the emergent vegetation is subjected to a dynamic wind loading, while the wind applies a shear on the water simultaneously subjecting the whole system to a wave-turbulent interaction. 
Hence, the first goal of the present work is to describe the onset and magnitude of wind-induced water flow in a standing water body in the presence of emergent vegetation with varying density and rigidity. To build a theoretical framework assisting future model development, a second goal is to delineate under what circumstances the wave and turbulence dominated regimes are separable so as to allow standard turbulence theory and standard linear wave theory to be applied at those decoupled regimes.

To address these goals and issues experimentally, Particle Imaging Velocimetry (PIV) experiments have been conducted in the laboratory to explore the characteristics of turbulence induced by wind shear on a static water body systematically for different water heights $(h)$ and mean wind speeds $\left(U_{a}\right)$ for each of the following scenarios: no vegetation, rigid sparse vegetation, rigid dense vegetation, flexible sparse vegetation and flexible dense vegetation. Analysis of the experimental data facilitates the understanding of the effects of $h, U_{a}$, vegetation flexibility and vegetation density, all of which are external conditions needed in describing the flow-dynamics within the water body. Another important aspect of the present attempt is that no instance of PIV experiments of such a type involving flexible moving canopy and wind on water have been found in the literature although PIV experiments with rigid vegetation and moving water flow have been conducted in the past as reviewed elsewhere (Nezu and Sanjou, 2008). It is demonstrated in the present work that such a PIV experiment is possible indeed with proper handling and choice of materials and methods.

\section{Experiment}

The PIV experiments were conducted in the Fluid Mechanics workshop at the Institute of Hydroscience and Engineering (IIHR) - University of Iowa. The dimensions of the flume (of width $35 \mathrm{~cm}$ ) can be found in panel (a) of figure 1. The wind was generated by a fan (with three preset wind speed settings) mounted above the flume. For the experimental runs with the canopy, nylon cable ties ( $4 \mathrm{~mm}$ wide, $1 \mathrm{~mm}$ thick) 'planted' on a test bed were used as a model vegetation. Two different vegetation densities, $\lambda_{d}=0.39$ for sparse and $\lambda_{d}=1.04$ for dense, were used. Full tie (or canopy) height $h_{c}=27.3 \mathrm{~cm}$ was used for flexible vegetation, while the same ties and same vegetation density cut to $h_{c}=7 \mathrm{~cm}$ represent the rigid vegetation (but $h<h_{c}$ in all experiments except the deepest water conditions for rigid cases). It is to be noted that the property of the nylon cable ties is such that when cut to a smaller length, they become quite rigid thus obviating the necessity to use other materials for stiff vegetation and reducing cost. Moreover, the rigid cases are submerged for deepest water conditions, however, they server the main purpose of applying drag for most part of the fluid, since the protrusion length does not matter in those cases. The $\lambda_{d}$ was calculated as $\lambda_{d}=n w_{t} h / s_{v}$ following Nezu and Sanjou (2008), where $n$ is number of cable ties, $w_{t}$ is the width of each tie, and $s_{v}$ is the test bed 'vegetated' area. The $h$ was used here in the estimation of $\lambda_{d}$ instead of $h_{c}$ because the vegetation was emergent for all runs as earlier noted. The selection of an optimal $\lambda_{d}$ is not trivial given that it is an optimization between maintaining realistic vegetation densities, as well as maintaining sufficient open area to allow particle imaging that can be challenging where the vegetation is flexible and oscillating. For the purpose of imaging, water was seeded with neutrally buoyant particles (spherical hollow glass spheres 110P8, Potters Industries) and illuminated with a laser light sheet passed through a thin $(\approx 1.5 \mathrm{~mm})$ slit carved on the test bed. A high frequency camera (Motion Xtra NX4-S1- Integrated Design Tools, Inc.) was used for imaging, and a sampling frequency of $30 \mathrm{~Hz}$ has been used. Each run was recorded for 30 seconds allowing enough full cycles of oscillations by the vegetation- thus generating 900 images for every run. The PIV 
data analysis was conducted using the open source software PIVLab (Thielicke and Stamhuis, 2010). Images for the experimental set-up and the test bed are shown in panels (b) and (c) of figure 1 . The $U_{a}$ was measured using a standard hand held anemometer approximately at $z_{a}=5$ $\mathrm{cm}$ above the water surface. The wave height was measured (although not synchronized with every experimental run) with a wave gauge (Kenek water level measuring system, servo type, SW-10.1). Wave frequencies $\left(f_{w}\right)$ were obtained by Fast Fourier Transforming (FFT) the time evolution of the water surface and choosing the dominant peak frequency. The measurements were conducted midway of the test bed so that the waves are fully developed and representative of the wave condition. The wavelength $\left(\lambda_{w}\right)$ was measured from visual analysis of the images of the water surface and one mean value was used. The wave celerity $\left(U_{w}\right)$ was determined by using $U_{w}=f_{w} \lambda_{w}$. The wave amplitude $\left(A_{w}\right)$ was measured from the average wave height collected in the water surface elevation data. The quantity $A_{w} / \lambda_{w}$, called wave steepness factor, was then computed. The wind and wave conditions are listed in the table 1:

Table 1: Wind and wave conditions sampled by the experiments showing wind speed $U_{a}$, wave frequency $f_{w}$, wavelength $\lambda_{w}$, wave velocity $U_{w}$, and wave amplitude $A_{w}$ (m).

\begin{tabular}{|l|l|l|ll|l|l|l|}
\hline \hline$U_{a}\left(\mathrm{~m} \mathrm{~s}^{-1}\right)$ & $f_{w}(\mathrm{~Hz})$ & $\lambda_{w}(\mathrm{~m})$ & $\begin{array}{l}U_{w} \\
\left.\mathrm{~s}^{-1}\right)\end{array}$ & $(\mathrm{m}$ & $A_{w}(\mathrm{~m})$ & $U_{w} / U_{a}$ & $A_{w} / \lambda_{w}$ \\
\hline $2.83(\mathrm{~W} 1)$ & 4.0 & 0.076 & 0.30 & & 0.00100 & 0.108 & 0.013 \\
$3.33(\mathrm{~W} 2)$ & 4.2 & 0.076 & 0.32 & & 0.00129 & 0.096 & 0.016 \\
$3.37(\mathrm{~W} 3)$ & 4.4 & 0.076 & 0.34 & & 0.00134 & 0.100 & 0.018 \\
\hline
\end{tabular}

The wave steepness factor does not exceed 0.05 in nature (Yang, 2001) and the experimental condition here is well below that limit. Also, the $U_{a}$ range does not vary appreciably, though all of the three selected $U_{a}$ are sufficiently large to generate turbulence in the water system. As shown later, these differences in $U_{a}$ also generate different stresses at the water surface due to the presence of emergent vegetation. Three $h$ values were used in the experiment and are designated as $\mathrm{H} 1, \mathrm{H} 2$ and $\mathrm{H} 3$ respectively, and for each $h$, three $U_{a}$ (and thus wave) conditions designated as W1, W2, and W3 were explored. Hence, different combinations of $h$ and $U_{a}$ are designated as $H i W j$, where $i=1,2,3$ and $j=1,2,3$. For each of the $9 H i W j$ combinations, 5 vegetation configurations were studied as earlier noted: no vegetation, sparse rigid, sparse flexible, dense rigid, and dense flexible. The water height characteristics are listed in the table 2. The classification of water depth used here matches the experimental condition in Yang (2001). The average wavelength is calculated based on linear wave theory according to the (implicit) formula (Yang, 2001)

$$
\lambda_{w}=\frac{g T_{w}{ }^{2}}{2 \pi} \tanh \left(\frac{2 \pi h}{\lambda_{w}}\right),
$$

where $T_{w}=1 / f_{w}$ is the wave period and $g$ is the gravitational acceleration. This computation $(=0.091 \mathrm{~m})$ overestimates the visually calculated wavelength of $0.076 \mathrm{~m}$ by about $18 \%$.

Given the small domain, lateral boundaries of the flume, and wind speed generation method, it is instructive to assess whether the experiments here are representative of large water bodies. To do so, $U_{a}$ in the absence of vegetation was used in combination with Charnock's equation (Charnock, 1955) to estimate the momentum roughness height $\left(z_{o}\right)$ and subsequent $A_{w}$ as well as the momentum flux at the air-water interface. Charnock's equation, valid for large fetch in the absence of waves generated from distant storms (or swells) and for stationary and planar 
Table 2: Water height conditions explored showing the measured water depth $h$ and the relative water depth $h / \lambda_{w}$ along with the designation used.

\begin{tabular}{|l|l|l|}
\hline \hline$h(\mathrm{~m})$ & $h / \lambda_{w}$ & Depth designation \\
\hline 0.125 & 1.64 & Deep (H1) \\
0.070 & 0.91 & Intermediate (H2) \\
0.025 & 0.32 & Shallow (H3) \\
\hline
\end{tabular}

homogeneous air flow well above the viscous or wave-induced roughness sublayer, is given as

$$
U_{a}(z)=\frac{u_{*}}{\kappa} \ln \left(\frac{z_{a}}{z_{o}}\right) ; z_{o}=0.011 \frac{u_{*}^{2}}{g},
$$

174

where $\kappa=0.4$ is the Von Kámán constant and $A_{w}=\alpha_{w} u_{*}^{2} / g$, with $\alpha_{w}$ being a proportionality constant depending on wavelength and steepness of waves being generated (Csanady, 2004). With $z_{a}=0.05 \mathrm{~m}$ (height of measurement- roughly the height of the center of the anemometer above the water surface) and measured $U_{a}$, the estimated air-side $u_{*}=(0.15,0.18,0.19) \mathrm{m} \mathrm{s}^{-1}$, resulting in $A_{w}=(0.9,1.3,1.4) \times 10^{-3} \mathrm{~m}$, reasonably close to the measured $A_{w}=(1.0,1.29,1.34) \times 10^{-3}$ m when $\alpha_{w}=0.4$. Adjusting by air and water densities, these air-side $u_{*}$ translate to water-side $u_{*}=(0.18,0.22,0.23) \times 10^{-3} \mathrm{~m} \mathrm{~s}^{-1}$ at the air-water interface. The horizontally averaged PIV measurements of $u_{*}$ near the water-air interface varied from 0.4 to $2.5 \times 10^{-3} \mathrm{~m} \mathrm{~s}^{-1}$ across all points near the surface suggesting that the Charnock's equation for the air-side is a lower-limit on such momentum transfer. This underestimation by Charnock's equation is partly due to the proximity of the $U_{a}$ measurements near the surface and partly due to the fact that the Reynolds stress is not constant near the water surface and the fan does not generate a classical mean logarithmic velocity variation with height as assumed by Charnock's equation. To illustrate the first point, estimated $A_{w}$ and $U_{a}$ measured at $z_{a}=0.05 \mathrm{~m}$ are used to compute the Keulegan number (wave height to viscous length scale) that varied from 10 to 15 in all cases, which is quite low for the application of Charnock's equation. Ideally, Charnock's equation applies in situations where the Keulegan number exceeds 100. Hence, the use of the 'rough-formulation' for inferring $u_{*}$ from $U_{a}(z)$ via Charnock's equation for $z_{o}$ is likely to be underestimated. Also, the fact that the stress is increasing in magnitude away from the interface (as discussed and shown later) suggests that the PIV measurements away from the interface may over estimate inter-facial values. Nevertheless, these calculations do suggest that the flume experiments 'mimic' key aspects of wind dynamics and inter-facial momentum transfer over large water bodies in nature despite the primitive wind generation mechanism and lateral edges of the flume.

\section{Data Analysis}

\subsection{General considerations}

Processing of PIV images produces velocity time series (both for the horizontal $u$ and vertical $w$ components) at every grid point that populate the region of observation (imaged by the high frequency camera, approximately $15 \mathrm{~cm} \times 15 \mathrm{~cm}$ ) except for those points obstructed for imaging by some moving element or the locations of the air region and test bed. These regions are blocked for the aid of image processing so that only the seeded fluid part of the image is analyzed. The grid size in the aforesaid observation window is $63 \times 63$ grid points consistent 
for all experimental runs. An instantaneous velocity field is depicted in figure 2 for the purpose of visualization. Rolling motions and reverse flow can be observed - providing a snapshot of the rich dynamics under consideration. Summary statistics for any flow variable are constructed by first time averaging at a point then horizontally averaging time-averaged quantities across the camera window field to produce profiles treated as space-time averaged quantities as common in canopy flow studies (Poggi et al., 2004; Raupach and Shaw, 1982). That is, instantaneous $u$ and $w$ at a point are first decomposed into a time-averaged and fluctuating components given as

$$
u=\bar{u}+u^{\prime} ; w=\bar{w}+w^{\prime},
$$

where over-bar denotes a time-averaged quantity and primes denote fluctuating quantity from time averages. The local stress $\overline{u^{\prime} w^{\prime}}$ and the longitudinal $\left(\sigma_{u}=\sqrt{\overline{{u^{\prime 2}}^{2}}}\right)$ and vertical $\left(\sigma_{w}=\sqrt{\overline{{w^{\prime 2}}^{2}}}\right)$ intensities can be computed at every grid point as well. Again, horizontal or line averaging is then applied over all time-averaged points to yield profiles of space-time averaged quantities (Poggi et al., 2004). These space-time averaged profiles are presented as a function of normalized height $z / h$, where $z$ is the distance from the flume bed. That is, $z / h=0$ is the channel bottom and $z / h \approx 1$ is the location of the mean air-water interface. For a given canopy configuration and $U_{a}$, these space-time averaged profiles are presented as a function of $z / h$ for the three $h$ scenarios to emphasize the (significant) effects of water depth on these space-time averaged flow statistics.

Because the flow field consists of simultaneous wave and turbulence effects, interpretation of the experimental data is subject to different choices. For example, Luhar et al. (2010) suggested a decomposition of the velocity data from a study of superimposed tidal flow and turbulence into a mean component (obtained by binning the data into different phases based on upward zero crossings and ensemble averaging the bins), a coherent wave component and turbulent fluctuations. However, since no prior knowledge is available about the nature of the flow field for the present problem from the literature, a logical choice would be examining the spectra and co-spectra of the co-existing wave and turbulence in the time series, which constitutes much of the material for this section. Indication of a near $-5 / 3$ power-law in the spectra can be treated as signature of fine-scaled turbulence presumed to be locally homogeneous and isotropic, while a flat spectrum would indicate unavoidable white noise. Any excitation close to the dominant wave frequency could indicate prevalence of wave dominance. Given that the noise content of PIV data can be high in such experimental configuration, identifying the noise component and removing it from the time series prior to any averaging (time and planar) becomes necessary.

In the spectral analysis here, $S_{x}(f)$ refers to the one-sided spectrum at frequency $f$ (in cycles per unit time or $\mathrm{Hz}$ ) of an arbitrary zero-mean stationary stochastic process $x(t)$ with variance $\sigma_{x}^{2}=\overline{x^{\prime 2}}=\int_{0}^{\infty} S_{x}(f) d f$. All spectra are first calculated at a grid point using Welch's averaged modified periodogram method with 4 sections and 50\% overlap, and with each section windowed with a Hamming shape and the computed periodograms for each window are then averaged. These spectra are then horizontally averaged for each $f$ to produce an ensemble $S_{x}(f, z)$. A similar procedure was used for all co-spectral calculations $S_{u w}(f)$, where $\overline{u^{\prime} w^{\prime}}=\int_{0}^{\infty} S_{u w}(f) d f$. For certain illustrations, the space-time averages - referred to as ensemble $S_{u}(f, z), S_{w}(f, z)$, and $S_{u w}(f, z)$ are compared at three typical $z / h$ labeled as top $(z / h=0.9)$, middle $(z / h=0.5)$ and bottom $(z / h=0.1)$, respectively.

Spectra and co-spectra for the no vegetation scenario (as reference case) and the flexible dense scenario are shown in figure 3 and figure 4, respectively. Panels (a), (d) and (g) present $S_{u}(f, z)$, panels (b), (e) and (h) present $S_{w}(f, z)$, and panels (c), (f) and (i) present $S_{u w}(f, z)$ at the three $z / h$ previously mentioned (top, middle, and bottom), respectively. In every panel, the 
spectra and co-spectra are plotted for all three $h$ cases - H1 (deep), H2 (intermediate) and H3 (shallow) to emphasize the role of $h$ at a given $z / h$. Only the highest $U_{a}$ (i.e. W3) is used for all these cases for the purpose of demonstration (and these cases are labeled as H1W3, H2W3, H3W3 in the figure legends). The $-5 / 3$ power-law is also presented in the panels featuring the $S_{u}(f, z)$ and $S_{w}(f, z)$ spectra to identify possible signatures of inertial subrange turbulence (if any). The following observations can be made from figure 3 and figure 4 :

- For the no vegetation scenario (in figure 3), the shallowest spectra (i.e. those associated with $\mathrm{H} 3 \mathrm{~W} 3$ ) do not follow the $-5 / 3$ power-law at any $z / h$, although this data appear to be contaminated by a large noise component. The deep $h$ case (i.e. H1W3) is also 'noisy' for large $z / h$, but shows the signatures of persistent turbulent structures with decreasing $z / h$ - nearly following a $-5 / 3$ power-law scaling at the middle and bottom $z / h$. The behavior of the intermediate $h$ case (i.e. H2W3) falls between the deep and shallow $h$ scenarios. This intermediate $h$ case also displays the existence of an inertial sub-range in the middle and bottom $z / h$ levels. Possible explanation for this behavior is that the flow near the airwater interface is subjected to extensive disturbances that prevent any inertial subrange to be resolved. Also, the temporal sampling (i.e. $30 \mathrm{~Hz}$ ) is too coarse to delineate a possible restricted inertial subrange followed by viscous dissipation range at high frequencies. This is analogous to spectra of turbulence near solid boundaries where the velocity spectra commonly lack extensive inertial subrange scales in the roughness (or buffer) regions. For the deep flow cases (i.e. H1W3), after turbulence is generated at the water-air interface, eddies get the allowance (higher $h$ ) to populate the full range of scales analogous to many shear flows when $h$ becomes sufficiently large. In sum, the spectra appear to share some analogies with other shear flows (e.g. an inverted boundary layer) in terms of expectations as to the onset of an inertial subrange, where the generation mechanism occurs at the air-water surface, and with sufficient distance from the air-water surface (i.e. decreasing $z / h)$, an inertial subrange forms provided $h$ is sufficiently large. There exists a peak in the $S_{w}(f)$ close to $4 \mathrm{~Hz}$, which is the dominant wave frequency, indicating the effects of the waves to be significant but only for the deepest $h$ (i.e. H1W3) thereby illustrating the role of $h$ at a given $z / h$. Interestingly, the wave effects are captured most significantly at the middle $z / h$. The fact that the vertical velocity spectra also capture the same wave effects demonstrates the existence of vertical wavy structures. A plausible explanation might be that the rolling motions (or orbitals) from the passage of the traveling waves move downwards from the air-water interface before being damped by the presence of turbulence close to the bottom. For the intermediate and shallow $h$ cases, the orbitals might also be appreciably distorted by the presence of strong turbulence as previously discussed. The fact that the wave amplitude appears to be most visible in the middle layers (of H1W3) differs appreciably from experiments conducted by wave-makers, where the wave generation frequency is significant at all depths.

- For the flexible dense scenario (in figure 4), all three $h$ cases of deep, intermediate and shallow closely follow the $-5 / 3$ power law behavior- indicating the possible presence of fine-scaled turbulence characterized by approximate inertial subrange scaling at all three $z / h$. This pattern is displayed by both $S_{u}(f, z)$ and $S_{w}(f, z)$. This observation is significant, indicating that the presence of flexible vegetation introduces new length scales by means of vegetative drag that then leads to fine-scaled turbulence. There also exists a peak in the spectra close to the $4 \mathrm{~Hz}$ wave frequency. However, contrary to the no vegetation scenario, 
the wave peaks are much more 'diffused' around the $4 \mathrm{~Hz}$ frequency and they can be observed at all $z / h$ and for all three $h$ cases - deep, intermediate and shallow. The impact of surface waviness is much enhanced due to a weakened turbulence by vegetative drag. Whether or not new frequency peaks are introduced due to the waviness of the vegetation can be another interesting issue.

- The co-spectra appear noisy in all cases for both no vegetation and flexible dense scenario (despite planar averaging). However, there is an indication of co-spectral activity (positive and negative) close to $4 \mathrm{~Hz}$ for the flexible dense scenarios, though the activity seems to average out in the range of $2-5 \mathrm{~Hz}$ (meaning no significant net momentum transfer due to the wave component).

As observed from this spectral analysis of the instantaneous velocity time series, wave and turbulence appear to be intertwined in a complex fashion even when the spectra are horizontally averaged. The noise content of the data appear high (and contaminates the co-spectra significantly). Hence, it becomes necessary to reduce the spectral signatures of the noise first. After cleaning the individual series from noise, it might be possible to separate out the wave and turbulence and discuss their bulk properties since there is a signature of a higher spectral energy density close to the dominant wave frequency.

\subsection{Separating signal from noise in Fourier domain}

To proceed with the exercise of separating the $u$ and $w$ time series into 'signal' and 'noise' - a Lorenz curve type analysis is performed. This curve was first proposed by the economist M.O. Lorenz in 1905 to quantify whether the imbalance in wealth distribution based on taxable income is increasing or decreasing in time (Lorenz, 1905). Since its inception, this curve has been extended to measure imbalances in energy distribution of any time series and in an arbitrary domain (e.g. time, Fourier, or wavelet) as discussed elsewhere (Katul and Vidakovic, 1998; Lorenz, 1905; Vidakovic, 2009). The basis of this approach is that the highly energetic events are clustered into few frequencies and once the frequency modes are sorted on their energetic basis, the high energy modes can be collected and translated back into the time domain to obtain the 'cleaned' signal. The rest of the frequencies corresponding to the lower energetic modes can be translated back to time domain to re-construct the noise time series thereby allowing a determination of the signal-to-noise ratio (SNR). To proceed with this approach, a 'cutoff' energy threshold needs to be established above which the energy would correspond to 'signal' and below which the energy would correspond to 'noise'. The theoretical basis for this threshold, which is based on the maximum curvature in the Lorenz curve, is discussed in Appendix A. Another important assumption in this approach is that the noise modifies the spectral energy content of a series but not the phase-angle (i.e. surrogate to time location of coherent energetic events). Constructing a Lorenz curve on the Fourier coefficients aids in establishing this threshold as has been discussed elsewhere (Katul and Vidakovic, 1996, 1998; Vidakovic, 2009). Further details about this filtering method can be found in the Appendix A. Only sample results of the outcome of the analysis are shown in figure 5, where the original $u$ time series, the Lorenz-cleaned and the noise series are displayed. The 'signal' and the 'noise', when summed reconstruct the original series (by definition) and is confirmed here by the zero residual between the reconstructed series and the original noise-infected time series.

To lend confidence in the aforesaid analysis, linear averaged (over all realizations at a particular height) spectral energy densities of the 'signal' and 'noise' should be constructed. The 
noise, if un-correlated or 'white', exhibits a flat spectrum that is most evident at high frequencies. Figure 6 shows spectra for the signal and the noise for the flexible dense scenario for all three different $h$ cases- deep (H1), intermediate (H2) and shallow (H3). Panels (a) - (b), (e) - (f) and (i) - (j) show the spectral energy density for signal and noise respectively for the $u$ at three different $z / h$ levels - top, middle and bottom. Panels (c) - (d), (g) - (h) and (k) - (l) show the spectral energy density for signal and noise respectively for the vertical velocity for the same experimental configuration and locations. As observed, the spectra for noise are commonly flat at all levels of the flow for both the $u$ (panels (b), (f) and (j)) and $w$ components (panels (d), (h) and (1)). This imparts some confidence to the filtering performed on the original time series given that the spectra of the noise were not previously assumed. On the other hand, signatures of $-5 / 3$ scaling behavior can be observed in the 'cleaned' signal both for the $u$ (panels (a), (e) and (i)) and $w$ (panels (c), (g) and (k)) spectra at all $z / h$ considered. With this separation between signal and noise, the SNR for different experimental runs can be estimated based on the lorenz-cleaned signal and noise variances. As per calculations, the SNR is about $3-4$, which is moderate but expected for PIV data in such a setting. The coefficient of determination $\left(R^{2}\right)$ between the signal and the noise components for all the experimental runs was also conducted suggesting that signal and noise are reasonably uncorrelated or weakly correlated. Interestingly, the $R^{2}$ values between the signal and noise are least for the deepest flow (H1) cases (0-0.15), increasing slightly with the shallower cases, $0-0.18$ for the intermediate cases $(\mathrm{H} 2)$ and $0-0.30$ for the most shallow $(\mathrm{H} 3)$ cases.

\subsection{Separating wave from turbulence in de-noised signals}

The waves, now visible in the de-noised series as peaks around the dominant wave frequency of $4 \mathrm{~Hz}$ in figure 6, have been determined from separate measurements. Instead of appearing as a spike at $4 \mathrm{~Hz}$, the peak in the de-noised series is partially diffused towards lower frequencies. Hence, a frequency bandwidth of $2-5 \mathrm{~Hz}$ can be selected and it can be assumed that any activity (or energy) within this bandwidth is wave induced. The rest of the series contains turbulent effects. Any other effect from the movement of vegetation might be included via another frequency window. If it is outside a clear window width, it might show up in the turbulent spectra because by then the wave energy has already been transferred to turbulent kinetic energy. To achieve this filtering, spectral energy of a series is first computed from the Fourier components and the part of the series contained in the specified frequency window. The spectral energy between the extremities of the frequency window is then linearly interpolated. Any peak above the interpolated energy is considered part of the wave component. The rest of the series is considered turbulent. Translating back the wave and turbulent components to time domain yields the now separated turbulent and wave series. The filtering approach is explained in Appendix B. Figure 7 shows ensemble averaged wave and turbulent spectra computed from the wave and turbulent series after they are separated from each other using the frequency filtering approach for the flexible dense scenario. Every panel display the spectral energy density for three depth cases, deep (H1) in black, intermediate (H2) in blue and shallow (H3) in red. Panels (a) and (b) show the wave and turbulence spectra respectively for the horizontal velocity and panels (c) and (d) show the wave and turbulence spectra for the vertical velocity for the top level of the flow depth for each cases. Panels (e) and (f) show the wave and turbulence spectra respectively for the horizontal velocity and panels (g) and (h) show the wave and turbulence spectra for the vertical velocity for the intermediate level of the flow depth for each cases. Similarly, panels (i) and (j) show the wave and turbulence spectra respectively for the horizontal velocity and panels (k) and (l) show the wave and turbulence spectra for the vertical velocity for the bottom level of the flow 
depth for each cases. Figure 8 represents the wave and turbulent spectra but for the no vegetation scenario. Similar figures for the flexible sparse scenario, rigid dense and rigid sparse scenarios were derived but are not shown for brevity. Key observations from these figures for the wave and turbulence are as follows: For the no vegetation scenario, the spectrum of wave always display a peak around $4 \mathrm{~Hz}$ as earlier noted. The peak is not a sharp one but is rather diffused towards lower frequencies. The wave spectra follows some scaling above $5 \mathrm{~Hz}$ (the top extremity of the frequency filtering window), but not below $2 \mathrm{~Hz}$ (the low end of the filtering window). This indicates that the wave component does not influence high frequency motion associated with fine-scaled turbulent eddies. However, the larger eddies comparable to the wave orbitals in size distort the wave motion and diffuse the wave energy towards lower frequency. Any other low frequency motion below $2 \mathrm{~Hz}$ display a noise-like flat spectrum. This phenomenon is true for all three $z / h$, for all three $h$ cases and for both $u$ and $w$. Similar behavior is exhibited by the flexible dense scenario. Hence, the fate of the wave motion due to interaction between wave and turbulence appears similar irrespective of presence of vegetation. However, they might vary in the energy content of the dominant wave modes.

The behavior of the turbulent motion varies from scenario to scenario as expected. In the no vegetation scenario, the turbulence close to the air-water interface is governed by detached eddies. This is indicated by a flat spectrum at the lower frequencies and a spectrum close to a -1 power law (the signature of which is often attributed to detached eddies or eddies much larger than the measurement height in classical boundary layers) at the higher frequencies for all three $h$ cases (Banerjee and Katul, 2013; Kader and Yaglom, 1991; Katul et al., 1996; Katul and Chu, 1998; Nikora, 1999; Perry and Chong, 1982). However, for $z / h<0.5$, the deep and intermediate $h$ cases (H1 and $\mathrm{H} 2$ ) become inertial, displaying a $-5 / 3$ scaling. The shallow $h$ case (H3) does not display such an inertial behavior. This can possibly be explained by the fact that for the shallow $h$ cases, turbulent eddies get distorted by the wave orbitals throughout all $z / h$ - whereas for the deep and intermediate $h$ cases, the wave orbitals do not directly distort the turbulent eddies for the deeper layers $z / h<0.5$. Also, the behavior of the $w$ spectrum is similar to the $u$ spectrum. For the flexible dense vegetation, the turbulence is much more structured - meaning here that power-laws are evident over a broad range of time scales in the spectral domain. Even close to the surface, all three different $h$ cases display $-5 / 3$ power law spectra apart from the middle and bottom $z / h$. This is due to the possibility that the vegetative drag produces fine-scaled turbulence following wake-production. There exist diffused peaks at low frequencies superimposed on the turbulent spectra. These peaks arise from the oscillation of the flexible vegetation by wind. For the flexible sparse vegetation, the turbulent spectra is less organized than the flexible dense vegetation. The spectra close to the surface do not display a $-5 / 3$ scaling, but rather one close to a -1 scaling for all three height cases. Close to the middle and bottom $z / h$, the spectra follows $-5 / 3$ scaling closely. It is also important to note that the shallow $h$ case (i.e. H3) appears more structured than its counterpart for the no vegetation scenario. This indicates the importance of vegetative drag in the generation of fine-scaled turbulence component. For the rigid dense vegetation, the wave spectra contains more energy for the shallow $h$ cases. The intermediate and bottom $z / h$ display $-5 / 3$ scaling- again indicating of the positive effect of vegetative drag on possible generation of fine-scaled turbulence. However, there are no additional peaks on the turbulent spectra- reflecting the effect of rigid vegetation- with almost no oscillation with the wind. The rigid sparse vegetation scenario does not differ much from the rigid dense scenario, but shows more structure (i.e. spectral power-laws) than the no vegetation counterpart. 


\section{Results and Discussion}

Following the analysis of the high frequency $u$ and $w$ time series, their de-noising, and their separation into wave and turbulence, the two study objectives can now be addressed using all the $9 \mathrm{HiWj}$ combinations for each of the 5 vegetation configurations. In particular, the effects of the 5 vegetation configurations on the space-time averaged mean flow, turbulence and wave energetics, momentum transport, and isotropy are presented and discussed. As the $u$ and $w$ series have already been de-noised using the Lorenz filtering approach previously discussed, and the signal component has been decomposed into wave and turbulence using spectral filtering analysis, standard Reynolds averaging can be attempted. Moreover, wave and turbulent components of intensity and stress can be separately computed. Analyzing the original signal is retained in the context of mean velocities as the series become automatically de-meaned during extraction of wave and turbulent components.

\subsection{Mean horizontal velocity}

Figure 9 displays spatially averaged (horizontally across every level) profiles of the the mean horizontal velocity $(\bar{U})$ obtained with time-averaged raw $u$. Every panel presents the horizontalaveraged $u$ for all five scenarios - namely no vegetation, rigid sparse, rigid dense, flexible sparse and flexible dense. Panels (a), (b) and (c) present the deep (H1), intermediate (H2) and shallow (H3) $h$ cases respectively for the slowest $U_{a}$ (W1). Panels (d), (e) and (f) present the deep (H1), intermediate (H2) and shallow (H3) $h$ cases respectively for the intermediate $U_{a}$ case (W2). Similarly, panels (g), (h) and (i) present the deep (H1), intermediate (H2) and shallow (H3) $h$ cases respectively for the fastest $U_{a}$ case (W3). As before, the normalized height $z / h$ is used in all data representation. Key observations from figure 9 are presented based on the $h$ scenario as follows:

- For deep $h$ flow (H1 cases) with no vegetation, the flow direction is windward at the top $(z / h \approx 1)$ and reversed near the bottom of channel, the inflection point being about $z / h=$ 0.5 for all $U_{a}$. With rigid sparse, rigid dense and flexible sparse vegetation, similar profiles as the no vegetation case are observed at all $U_{a}$. With flexible dense vegetation and with increasing $U_{a}$, the reverse flow diminishes and at the highest $U_{a}$, the whole flow becomes windward in direction.

- For intermediate flow (H2 cases), the flow dynamics become richer. With no vegetation, the flow remains windward at the top and reverse at the bottom, the inflection point about $z / h=0.5$ at all $U_{a}$ as the $\mathrm{H} 1$ cases. With rigid sparse vegetation and with increasing $U_{a}$, the reverse flow diminishes and at highest $U_{a}$, the whole flow becomes windward as in the $\mathrm{H} 1$ cases. With rigid dense vegetation, the flow is strongly forward at all $U_{a}$. With flexible sparse vegetation, oscillatory and almost stationary flow is observed in the upper layers $(z / h>0.7)$ and uniform forward flow is noticed at the bottom. With flexible dense vegetation, the whole flow is forward at all $U_{a}$ as before.

- For shallow flow (H3 cases), the whole flow is windward or forward at all $U_{a}$ in all scenarios.

As observed, the presence of reversed flow is an important feature in wind shear induced turbulent flow. The existence of counter-current flow in the bottom layers has been observed by other studies as earlier discussed in the Introduction section (Tsanis and Leutheusser, 1988; Tsanis, 
1989). However, the effect of $U_{a}, h$ and vegetative drag or flexibility renders the dynamics more interesting. Irrespective of the presence of vegetation, as the flow becomes shallow (i.e. H3), the flow appears forward in the direction of $U_{a}$. In deep flow (i.e. H1), a high drag and flexible vegetation resists the counter-current movement at the bottom regions and makes the whole flow forward. At intermediate flow depth (i.e. H2), a similar effect of vegetation density is present as in the deep case, with the difference that the whole flow can become forward with a higher $U_{a}$. Figure 10 summarizes the key observations regarding the mean flow for all the experiments in the form of a binary phase diagram. Panel (a) summarizes the results for the no vegetation scenario, panel (b) for rigid sparse, panel (c) for rigid dense, panel (d) for flexible sparse and panel (e) for flexible dense scenario. A value of 1 in this presentation indicates fully forward flow and a value of 0 indicates existence of a counter-current flow at some location. The transition from one type of flow to another is represented by a number in between 0 and 1 . The switching from one type of flow to another is thus visible at all 9 different combinations of $h$ and $U_{a}$. It is interesting to note that the regions and extent of switching are different in every vegetation configuration, thus delineating the effect of vegetation flexibility and density in a clearer fashion on the onset of reversed flow. It is worth remembering here that the counter-current flow and the fully forward flow are both manifestations of local dynamics in the field of view of the PIV. The countercurrent flow is caused due to raising the water surface downstream and lowering the water surface upstream by the wind generated waves (which is called wind set-up) (Tsanis, 1989). In these cases, the effect of the interface boundary condition penetrates the water surface to a certain depth, below which the water flows in the reverse direction to maintain continuity in a vertical plane. While it is true that the experiments were conducted in standing water, it is important to attribute the counter-current flow at the bottom and fully forward flow to local effects within the window imaged by the PIV - rather than a consequence of a closed channel (i.e. global). Mathematically, the integrated continuity equation across any vertical section must be zero in the PIV imaged section for both counter-current and fully forward flow. That is, integrating $<\int_{0}^{h}(\partial U / \partial x) d z>+<\int_{0}^{h}(\partial W / \partial z) d z>=<\int_{0}^{h}(\partial U / \partial x) d z>+<W(h)>-<W(0)>=0$ (where angular brackets indicate line averaged quantities). If the difference between $W(h)$ and $W(0)$ is zero (as confirmed from analyzing the data), the depth integrated $\langle d U / d x\rangle$ term is zero, which implies that while the horizontal velocity might not be zero locally, its longitudinal gradient could be ensuring that continuity is satisfied within the imaged section. This assumption cannot be true everywhere locally - for example $<\int_{0}^{h} \partial U / \partial x d z>$ at the boundary upstream of the channel is positive and the $<\int_{0}^{h} \partial U / \partial x d z>$ at the downstream end is negative due to the bound nature of the flow. Hence, the cases where the whole flow moves forward is a result of the effect of a strong boundary condition at the air-water interface penetrating the whole flow depth, so the question of reverse gradient to maintain continuity does not arise in the PIV field of view. Vegetative drag also influences this dynamics. It is also to be noted that the the existence of reflected reverse flow generated at the closed boundaries in the close channel does not modify the results since the length and time scale of the observed dynamics are much smaller and well separated from the reflected flow. However, whether the manifestation of reflected flow is observed in cross stream flow can not be delineated from the two dimensional images and will be investigated in future campaigns.

\subsection{Momentum fluxes}

The effects of turbulence and wave on momentum flux are computed from the turbulent and the wave time-series, separately. For reference, the momentum flux obtained from the raw PIV 
measurements are also shown and discussed. Figure 11 presents the horizontally averaged turbulent stress $\left(\overline{u^{\prime} w^{\prime}}\right)$ profiles using the original series (i.e. includes wave and turbulence). As is the case for the mean flow analysis, every panel presents the stress for all five scenarios - no vegetation, rigid sparse, rigid dense, flexible sparse and flexible dense. Panels (a), (b) and (c) present the deep (H1), intermediate (H2) and shallow (H3) $h$ cases respectively for the slowest $U_{a}$ case (W1). Panels (d), (e) and (f) present the deep (H1), intermediate (H2) and shallow (H3) $h$ cases respectively for the intermediate $U_{a}$ case (W2). Similarly, panels (g), (h) and (i) present the deep (H1), intermediate (H2) and shallow (H3) $h$ cases respectively for the fastest $U_{a}$ (W3). Figure 12 and figure 13 represent the stresses computed separately from the turbulent and wave series. As observed from figure 11, the total stress (wave and turbulence) is small at the surface $(z / h=1)$, increasing gradually with decreasing $z / h$, but then diminishing again near the bottom $(z / h=0)$. The shape of these non-monotonic stress profiles provide a window to the mechanism of momentum transfer inside the water body. Momentum is transferred downward from the surface while being attenuated inside the water body, resulting in a small bottom stress. In the presence of vegetation, this attenuation is rather severe, resulting in the fact the the Reynolds stress in the no vegetation scenario is significantly larger than the other scenarios with vegetation. In some cases, there exists positive values of total stress close to the bed. The figures clearly suggest that turbulent stress is larger than the wave stress by an order of magnitude. Also, the wave stress is generally larger at the surface, diminishing steadily inside the water body with decreasing $z / h$, while the turbulent stress is largest in the middle of the flow $(z / h=0.5)$. This implies that the wave component of momentum is injected at the surface originating from the impinging effect of the wave orbitals. The turbulent component of the momentum, on the other hand, is maximum inside the water body, probably generated from the Kelvin- Helmholtz instability that produces rolling motions inside the water body roughly collocated with the inflection points in the mean velocity profile. Wakes generated in the inter-vegetation spaces contribute to momentum transfer; however, the measurements being two dimensional, these structures cannot be resolved in this experiment and require three dimensional stereo-PIV measurements.

\subsection{Turbulent and wave intensity}

Apart from the momentum transfer, the origin and transfer mechanism of the turbulent and wave energies are now discussed. Since the wave and turbulent series are already separated, it is possible to compare their respective intensities (called $\sigma_{u, T}$ for turbulent and $\sigma_{u, W}$ for wave, respectively). Figure 14 shows the relative importance of $\sigma_{u, W}$ with respect to $\sigma_{u, T}$ across all $z / h$ and for each case in the same arrangement. Key features of the turbulent and wave intensities and their relative characteristics are as follows:

- For most of the cases, $\sigma_{u, T}$ is maximum at $z / h=1$ and it reduces almost monotonically with decreasing $z / h$.

- For the deep $h$ case $(\mathrm{H} 1), \sigma_{u, T}$ is maximum for the rigid sparse scenario, followed by the rigid dense scenario, the no vegetation scenario, the flexible sparse scenario and the flexible dense scenario, respectively. It is clear that addition of flexibility and density increases sheltering. For the intermediate $h$ case $(\mathrm{H} 2)$, the no vegetation case registers maximum $\sigma_{u, T}$. For the shallowest $h$ case $(\mathrm{H} 3)$, this pattern is less clear but the flexible dense scenario always corresponds to the minimum $\sigma_{u, T}$, i.e., maximum sheltering.

- The wave intensity $\left(\sigma_{u, W}\right)$ shows consistent profiles for all the scenarios suggestive of a possible similarity solution to its behavior in terms of $z / h$. However, the wave intensity 
is generally less than the turbulent intensity. Another important distinction is that for the deepest $h$ case (H1), the wave intensity is maximum slightly below the water surface, and it tends to decrease towards the air water interface $(z / h=1)$.

- The turbulent intensity is generally greater than the wave intensity (about twice), except in the highest $U_{a}$ (i.e. W3) and for the deep $h(\mathrm{H} 1)$ cases. For the deep (H1) and intermediate (H2) $h$ cases, the turbulent intensity is much higher comparative to the wave intensity close to the bottom and not so much close to the top. On the other hand, for the shallowest case (H3), the relative importance of the turbulent and wave intensities are roughly consistent across $z / h$. This indicates that for the deep and intermediate $h$ cases, the turbulence effects penetrate much more in $z / h$ than the concomitant wave effects. However, for the shallow case, both the turbulence and wave effects penetrate the entire $z / h$ domain although the turbulent intensities remain higher than their wave counterpart.

\subsection{Isotropy}

The discussion on turbulent energetics invites the followup question regarding the isotropic (or lack of) nature in such a complex flow. To examine isotropic behavior in bulk flow statistics, Figure 15 shows a one to one comparison between the root-mean-squared (rms) component of the horizontal $\left(\sigma_{u}\right)$ and vertical velocity $\left(\sigma_{w}\right)$ horizontally averaged series. Panel (a) uses the raw velocity directly obtained from PIV analysis in the comparisons. Panel (b) uses the series after filtering out the noise, and panel (c) uses the separated turbulent from wave series and panel (d) uses the separated wave series. In all cases, the majority of the experimental runs show a near one to one behavior - indicating quasi-isotropic turbulent characteristics. This finding is relevant to operational models of turbulent dispersion noted in the introduction section, which require computation of turbulent diffusivity across different directions. For example, the horizontal and vertical turbulent diffusivity may be formulated as $K_{x}(z) \sim \sigma_{u}(z)^{2} \tau(z)$ and $K_{z}(z) \sim \sigma_{w}(z)^{2} \tau(z)$, where $\tau(z)$ is a relaxation time scale of the flow determined from the turbulent kinetic energy and its mean dissipation rate. Quasi-isotropic turbulent rms implies that $K_{x}(z)=K_{z}(z)$, which is a practical simplification when modeling turbulence in such a complex configuration.

\section{Summary and Conclusions}

The wind induced wave-turbulence interaction in the presence of rigid and flexible vegetation for different water heights (deep, intermediate and shallow) and different wind speeds (slow, medium and fast, although not varying widely) under five different scenarios-no vegetation, rigid sparse vegetation, rigid dense vegetation, flexible sparse vegetation and flexible dense vegetation was explored. Particle Imaging Velocimetry (PIV) experiments have been conducted and spectral analysis have been performed on the measured velocity time series to separate wave and turbulence effects on energy and momentum transfer under the aforementioned conditions. A Lorenz curve analysis has been performed on the raw velocity data to identify and filter out the noise. Surface wave characteristics are measured separately to identify the dominant wave frequency where it exists. The de-noised velocity component series are then analyzed in the frequency domain to identify wave activities around the dominant wave frequency to separate out the the wave and turbulent components of the signal. The no-vegetation scenario is found to be analogous to an inverted boundary layer flow where turbulence is generated in the air-water interface region and an inertial sub-range can be found deeper into the flow provided the flow depth is sufficiently high. On the other hand, the wave effects originate from the impinging motion of the rolling 
orbitals that move downward, resulting in higher activity close to the air-water interface before getting damped by the turbulent effects deeper inside the flow. Presence of flexible dense vegetation enhances the generation of fine scaled inertial turbulence by introducing new length scales via vegetative drag. The oscillation of the vegetation also diffuses the wave activity beyond the dominant wave frequency range. Another important feature of the coupled wind-wave-turbulent flow problem is the existence of counter-current mean flow in case of large flow depth. However, the flow can switch to a fully-forward one when reducing flow depth, or with increasing vegetative drag or flexibility under higher wind speeds. After a critical shallow flow depth, the mean flow is always in the direction of the wind flow. In terms of momentum transfer, significant difference is found to exist between the wave component of momentum flux and the turbulent component of momentum flux. The wave momentum flux is injected from the surface and attenuated monotonically deeper inside the flow, while its turbulent counterpart is maximum at the middle of the flow depth and highly attenuated close to the bed in the presence of vegetation. In terms of intensity, the wave intensity is generally less than the turbulent intensity although the turbulent intensity penetrates deeper than wave intensity inside the water for deeper flows. In shallow flows, both components coexist throughout the depth. It is also important to note that the vertical velocity components tend to exhibit similar energetics and momentum transfer mechanisms, indicating a quasi-isotropic nature. Future efforts will attempt capturing such a complex interaction by constructing numerical models of increasing detail and further experimentation involving turbulence measurements in air and across the water flow.

\section{Acknowledgments}

T.B. and G.K. acknowledge support from the National Science Foundation (NSF-EAR-1013339, NSF-AGS-1102227), the U.S. Department of Agriculture (2011-67003-30222), the U.S. Department of Energy (DOE) through the office of Biological and Environmental Research (BER)Terrestrial Ecosystem Science (TES) Program (DE-SC0006967), and the Binational Agricultural Research and Development (BARD) Fund (IS-4374-11C). The authors also acknowledge James Buchholz, $\mathrm{Ph} . \mathrm{D}$. and the staff at the Fluid Mechanics workshop, IIHR, University of Iowa for support while conducting the experiments. 
(a)

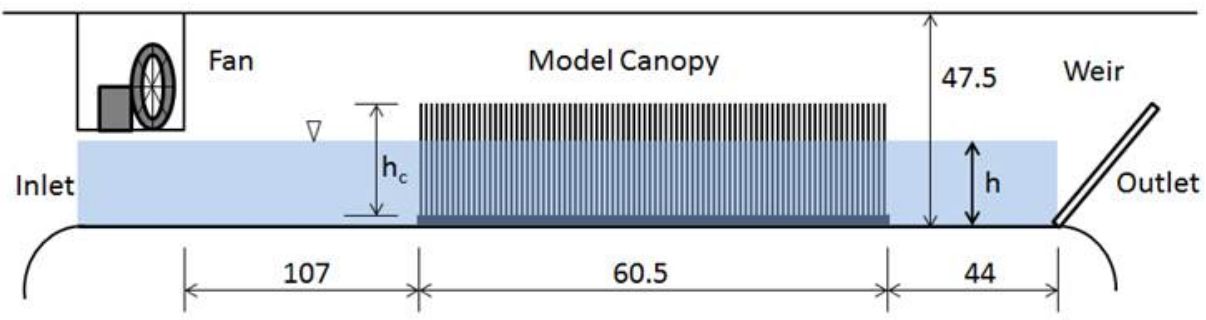

(b)

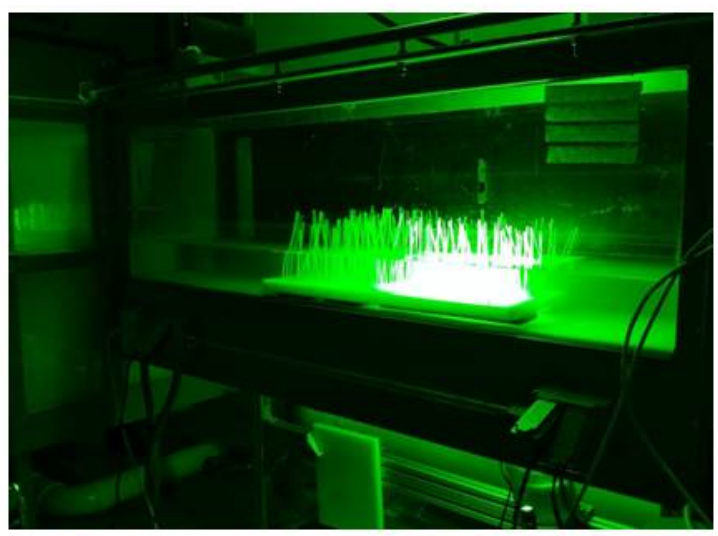

(c)

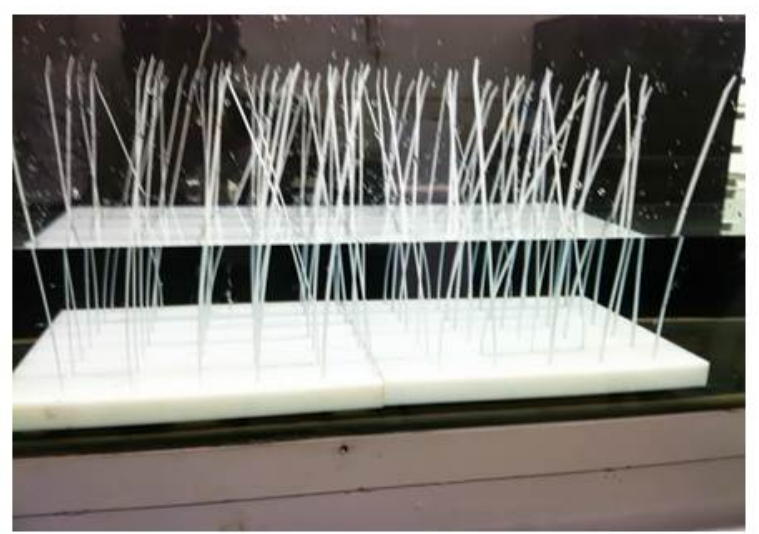

Figure 1: (a) Schematic diagram of the flume used for the experimental runs. All dimensions are in $\mathrm{cm}$. The width of the flume is $35 \mathrm{~cm}$. (b) Experimental set up, water seeded and illuminated by laser. (c) Test bed with model vegetation. 


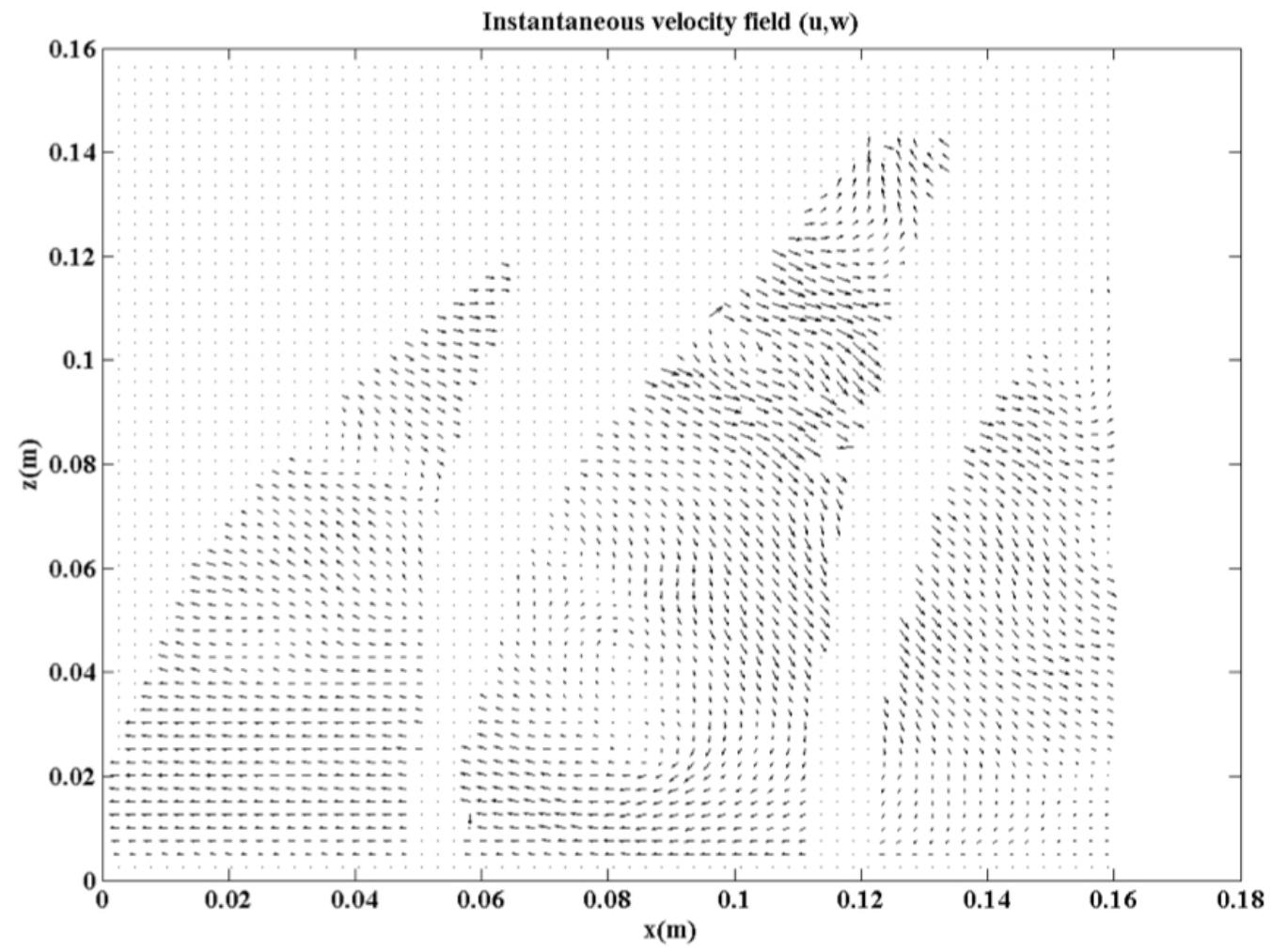

Figure 2: Instantaneous snap-shot of the two dimensional velocity field for the flexible sparse scenario, deepest water level (H1), and slowest wind speed (W1) termed as H1W1. The arrow size reflects the instantaneous velocity magnitude at the 64 x 64 grid points within a single image. 

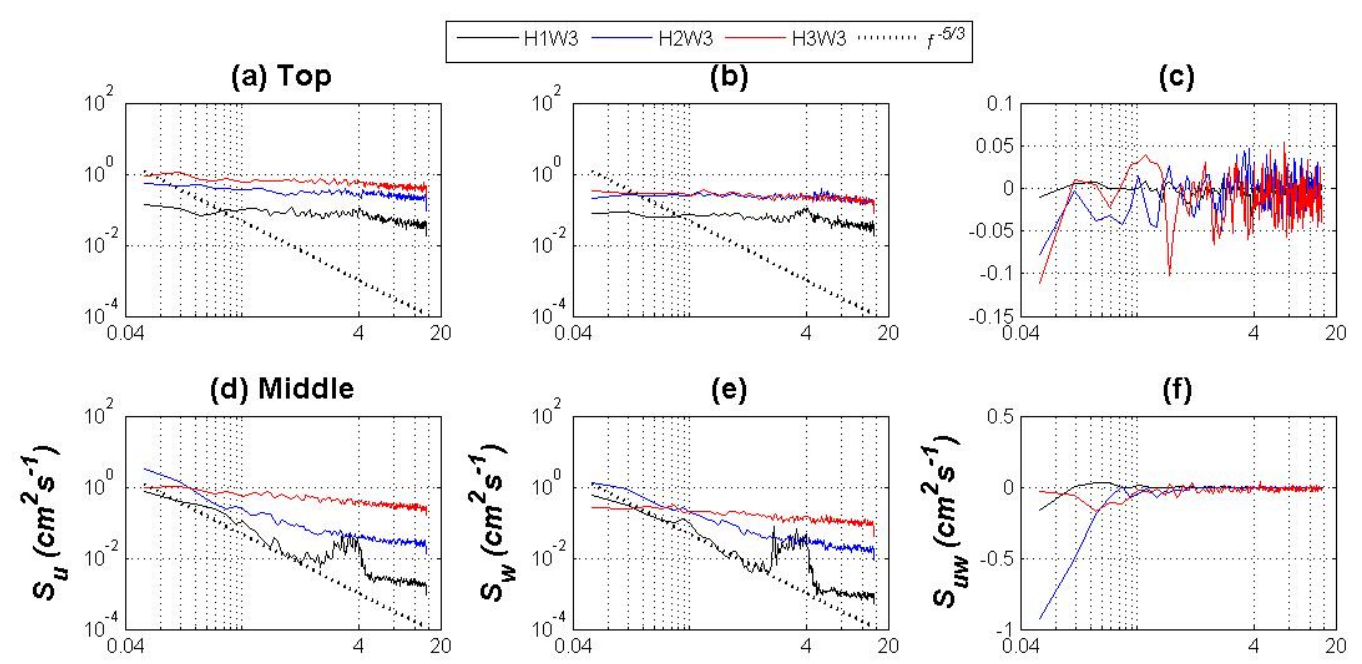

(c)
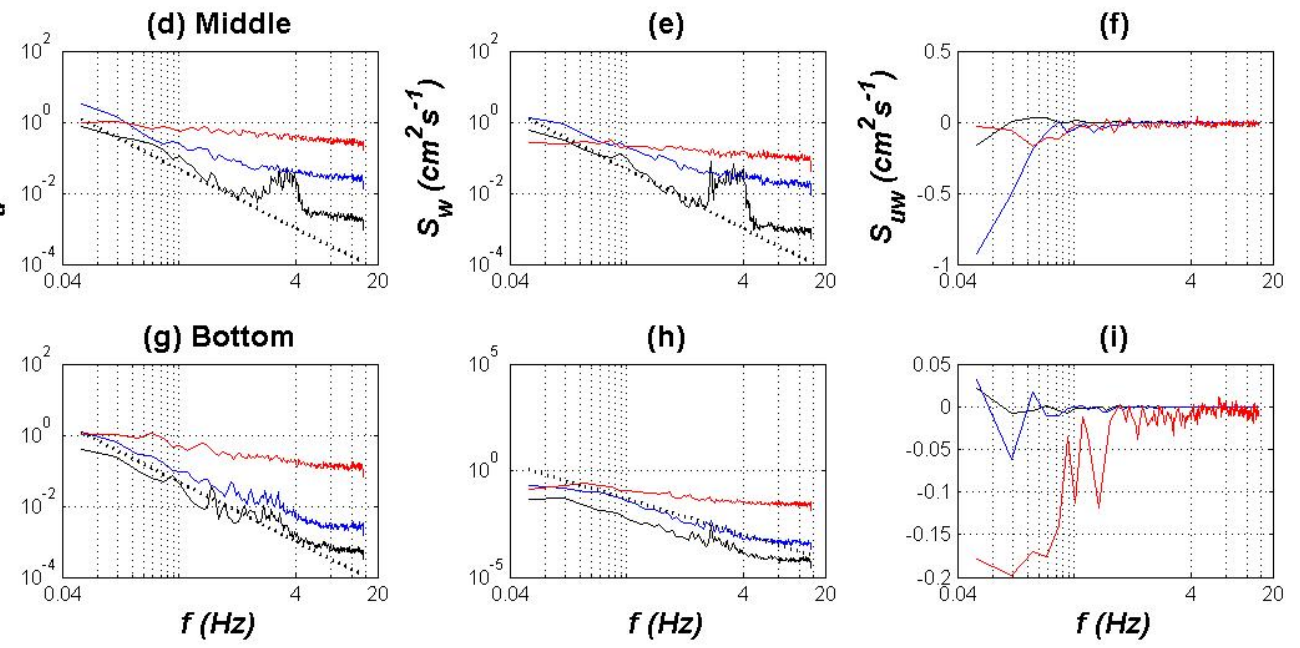

Figure 3: Spectra and co-spectra for the no vegetation scenario as a function of frequency $f$ computed from the raw time series. Panels (a), (d) and (g) represent the horizontally averaged spectral energy density for $u$ $\left(S_{u}(f)\right)$ and for three different $z / h$ - top, middle, and bottom, respectively. Similarly, panels (b), (e) and (h) represent the spectral energy density for $w\left(S_{w}(f)\right)$ at the same three $z / h$ and panels (c), (f) and (i) represent the co-spectra $\left(S_{u w}(f)\right)$ for the same three depths $z / h$. In every panel, the spectra and co-spectra are plotted for all three $h$ cases - H1 (deep - in black), H2 (intermediate - in blue) and H3 (shallow - in red) for the highest $U_{a}$ (i.e. W3). The $-5 / 3$ power-law is also plotted in a black dotted line in panels representing $S_{u}(f)$ and $S_{w}(f)$ spectra to locate signatures of inertial subrange turbulence. 
(a) Top

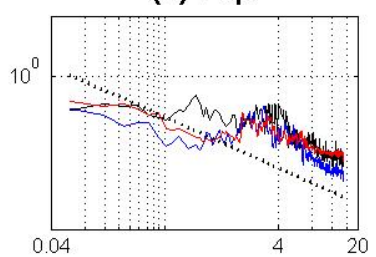

(d) Middle

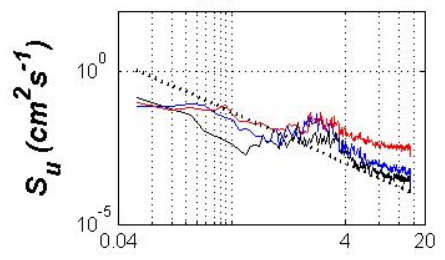

(g) Bottom

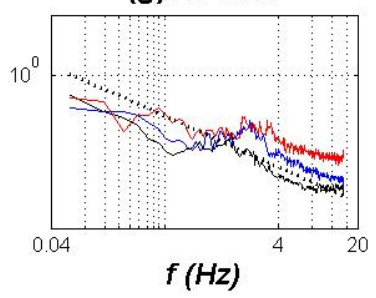

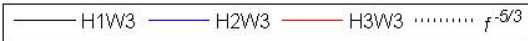

(b)

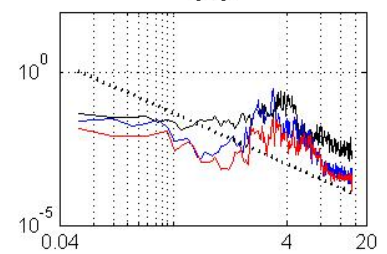

(e)

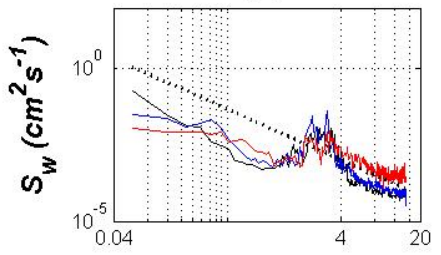

(h)

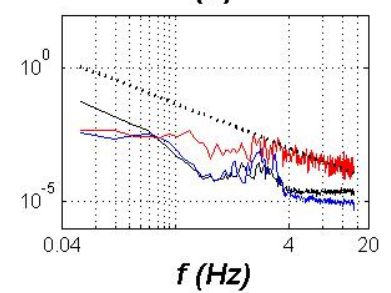

(c)

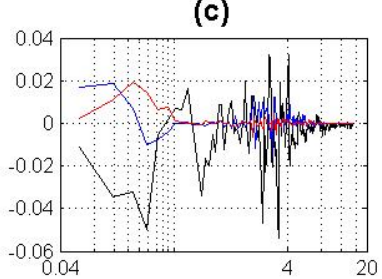

(f)

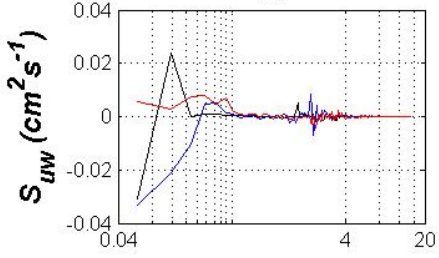

(i)

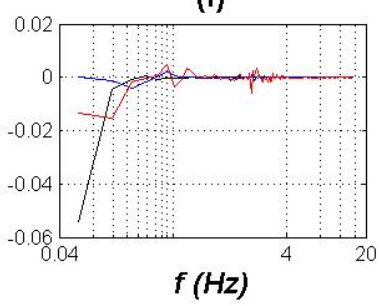

Figure 4: Same as Figure 3 but for the flexible dense vegetation scenario. 


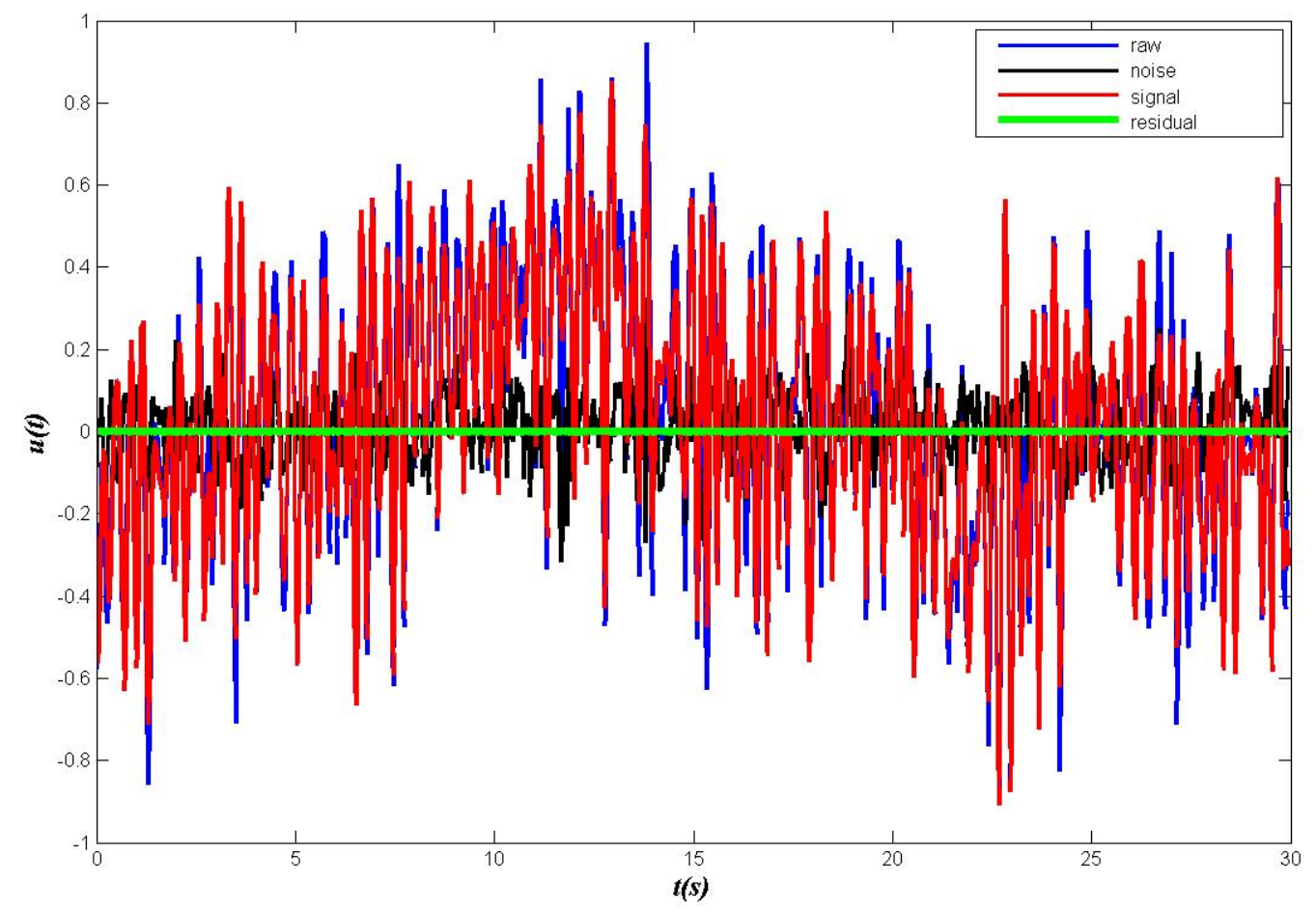

Figure 5: Sample time series for the flexible dense scenario for the deepest water- highest wind case (H1W3) demonstrating the decomposition of a velocity into 'signal' and 'noise' using spectral analysis and Lorenzcurve filtering. The series in blue color represents the original velocity time series $\left(\mathrm{cm} \mathrm{s}^{-1}\right)$. The series in red color represents the signal (the high energy component) and the series in black color represents the noise. The signal and the noise, when summed reconstruct the original series, indicated by a zero residual from the raw time series and is plotted in green color. Also it is noted that the coefficient of determination between the signal and the noise is almost zero- indicating they are completely uncorrelated. 
(a) Top

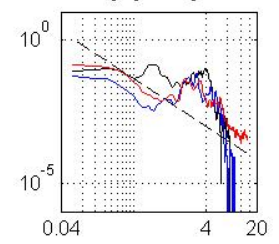

(e) Middle

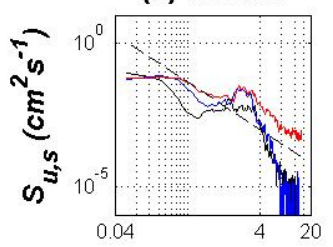

(i) Bottom

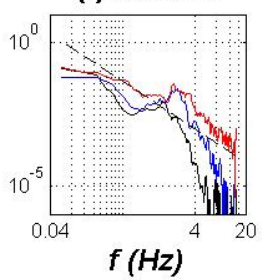

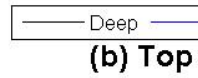

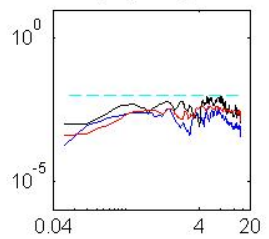

(f) Middle

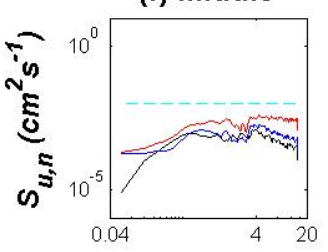

(j) Bottom

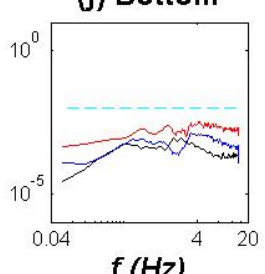

(c) Top

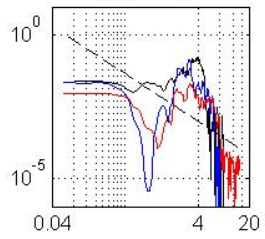

(g) Middle

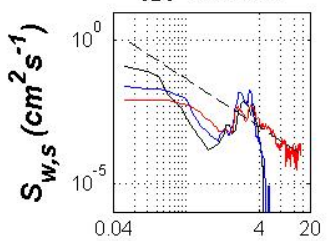

(k) Bottom

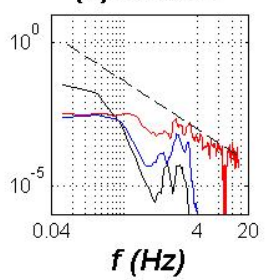

(d) Top

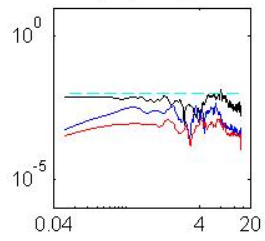

(h) Middle

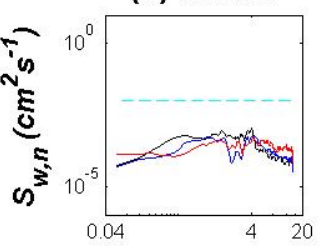

(I) Bottom

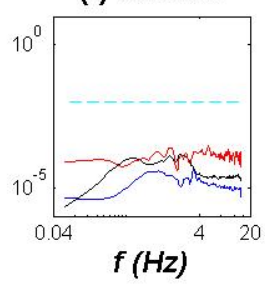

Figure 6: Horizontally averaged spectra for the signal $\left(S_{u, s}\right)$ and the noise $\left(S_{u, n}\right)$ for the flexible dense scenario for all three different $h$ cases- deep (H1), intermediate (H2) and shallow (H3) cases. Panels (a) - (b), (e) (f) and (i) - (j) show the spectral energy density for signal and noise respectively for $u$ at three $z / h$ as before - top, middle, and bottom. Panels (c) - (d), (g) - (h) and (k) - (l) show the spectral energy density for the signal and noise respectively for $w$ at the same three $z / h$. As observed, the spectra for noise is almost flat (shown by cyan dashed line) at all levels of the flow for both $u$ (panels (b), (f) and (j)) and $w$ (panels (d), (h) and (1)). 
(a) Top

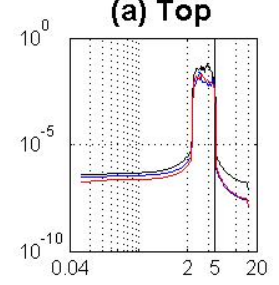

(e) Middle
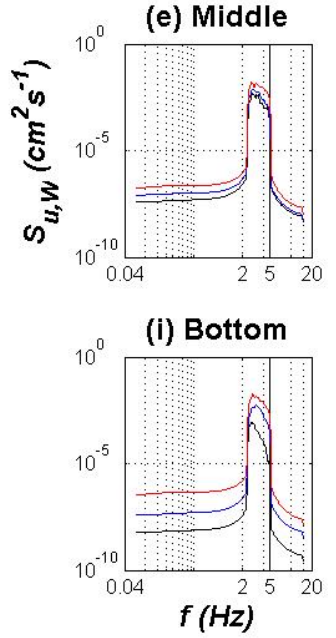
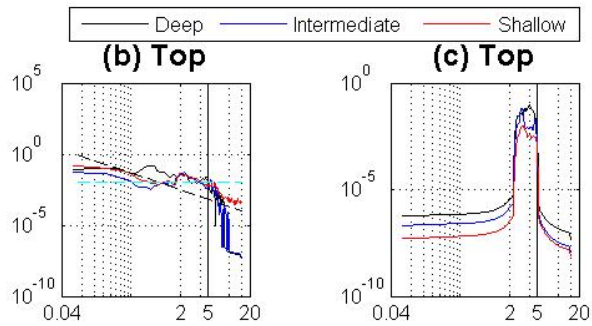

(g) Middle
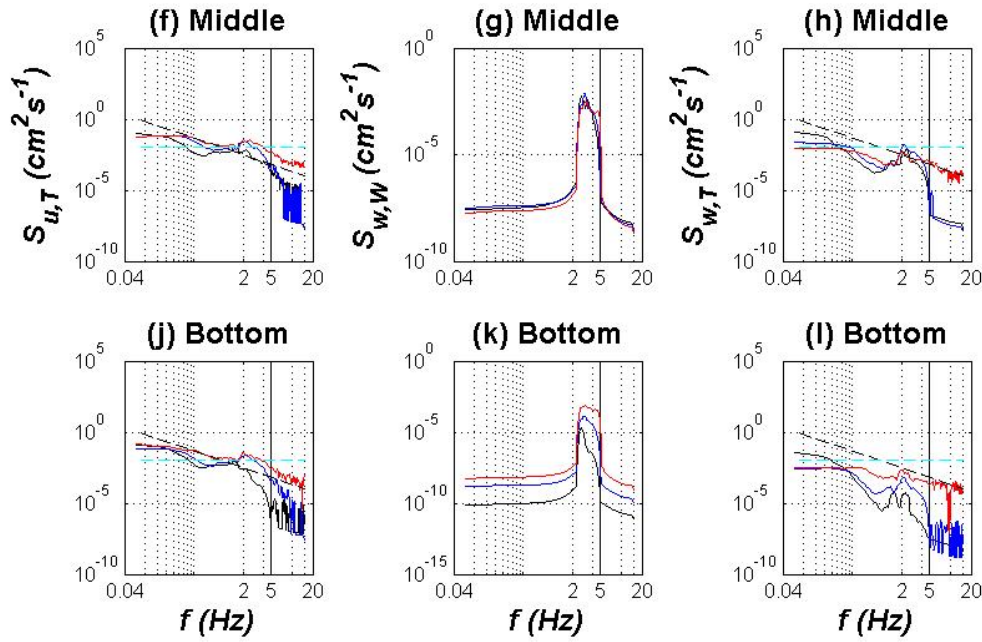

(k) Bottom

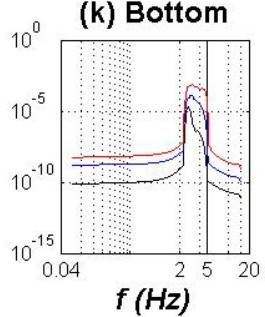

(d) Top
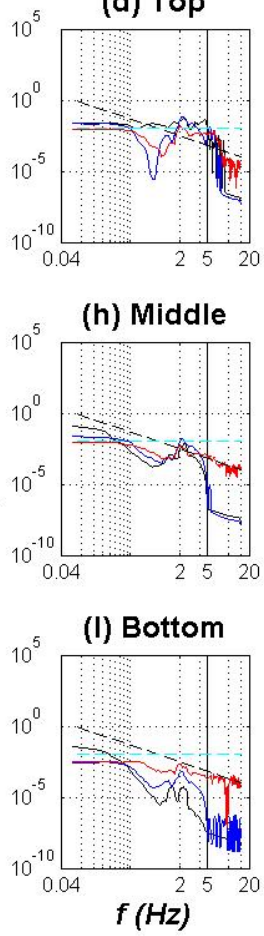

Figure 7: Horizontally averaged wave $S_{x, W}$ and turbulent $S_{x, T}$ spectra computed from the wave and turbulent time series after they are separated from each other using the frequency filtering approach for the flexible dense scenario. Every panel display the spectral energy density for three $h$ cases, deep (H1) in black, intermediate (H2) in blue, and shallow (H3) in red. Panels (a) and (b) show the wave and turbulence spectra respectively for $x=u$ and panels (c) and (d) show the wave and turbulence spectra for $x=w$ for the three $h$ cases and the top $z / h$. Panels (e) and (f) show the wave and turbulence spectra respectively for $x=u$ and panels (g) and (h) show the wave and turbulence spectra for $x=w$ for the intermediate $z / h$. Similarly, panels (i) and (j) show the wave and turbulence spectra respectively for $x=u$ and panels (k) and (l) show the wave and turbulence spectra for $w$ for the bottom $z / h$ for all three $h$ cases. 
(a) Top

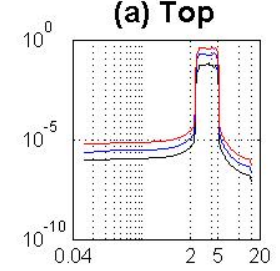

(e) Middle
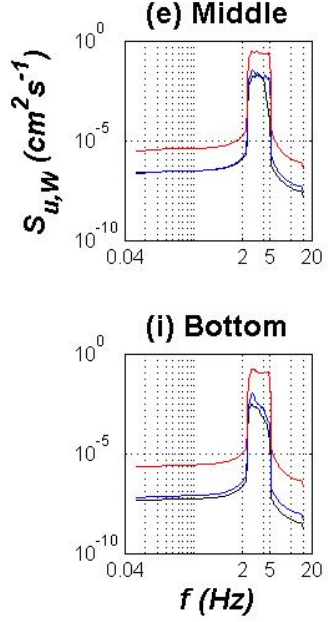

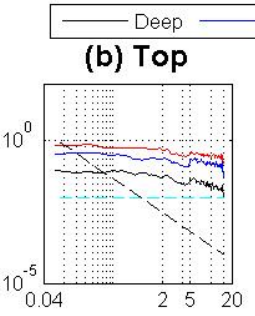

Intermediate

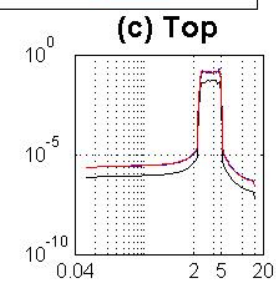

(f) Middle
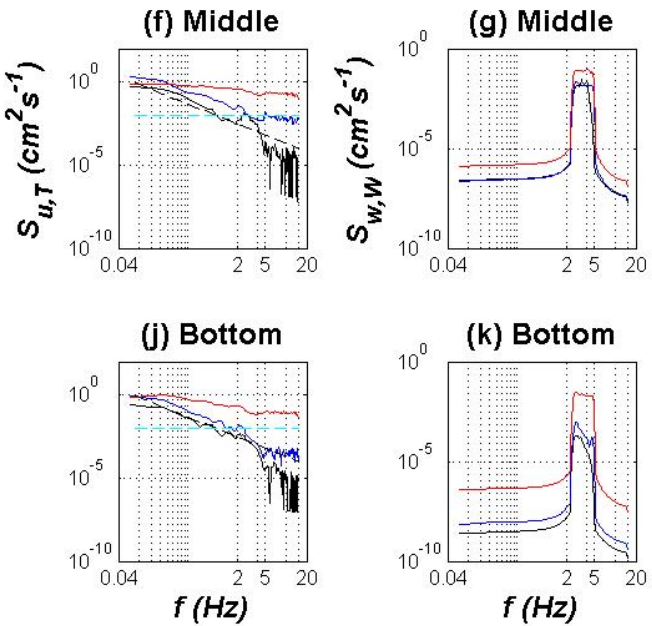

(d) Top

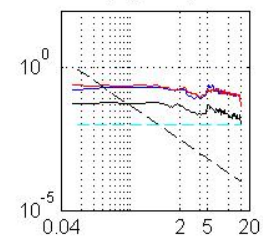

(h) Middle
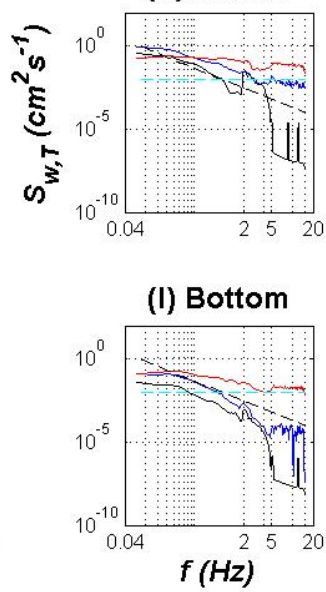

Figure 8: Same as Figure 7 but for the no vegetation scenario. 


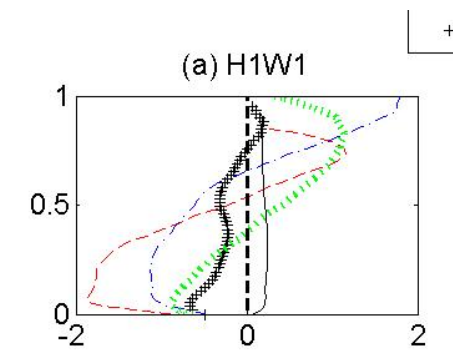

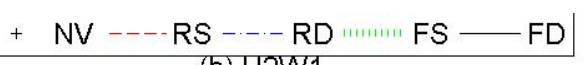
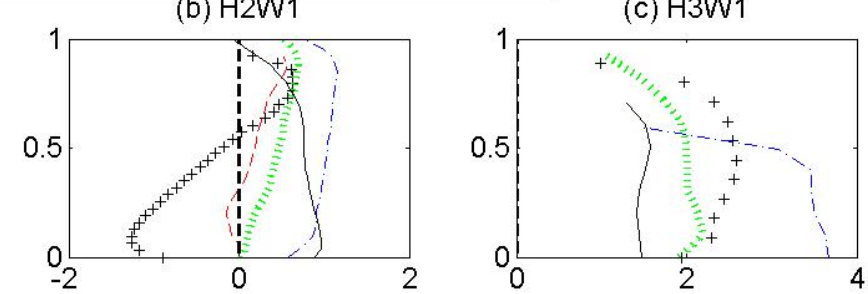

(d) H1W2

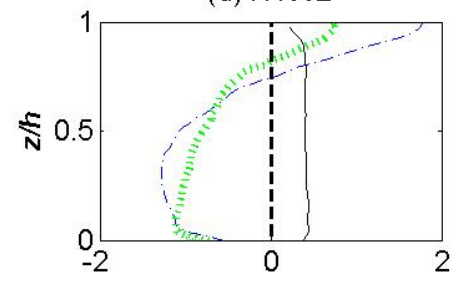

(e) $\mathrm{H} 2 \mathrm{~W} 2$

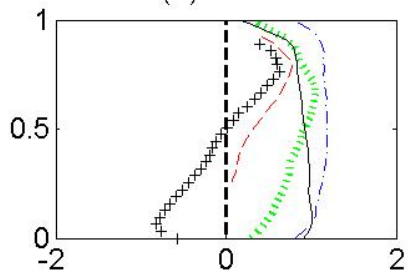

(f) $\mathrm{H} 3 \mathrm{~W} 2$

(g) $\mathrm{H} 1 \mathrm{~W} 3$

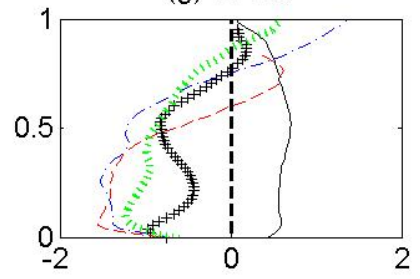

(h) $\mathrm{H} 2 \mathrm{~W} 3$
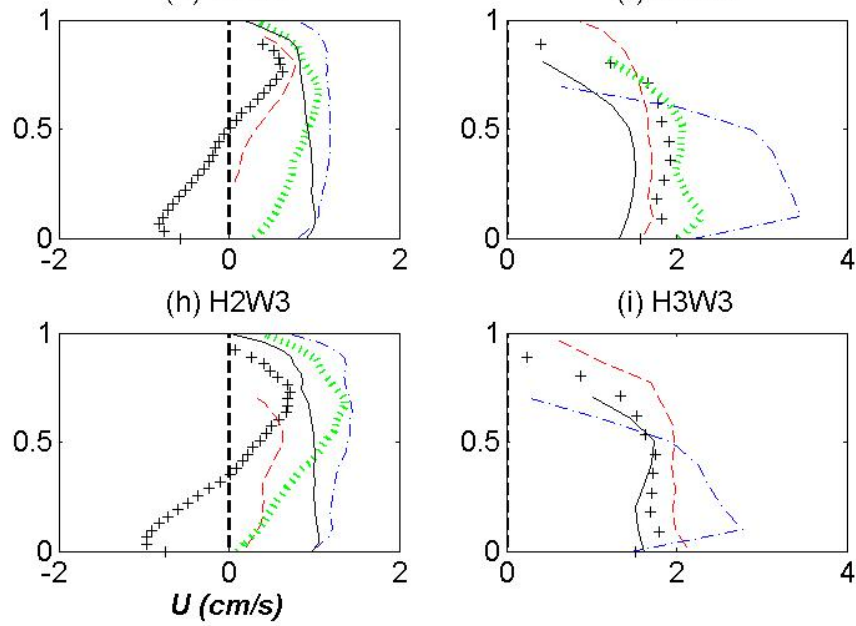

(i) $\mathrm{H} 3 \mathrm{~W} 3$

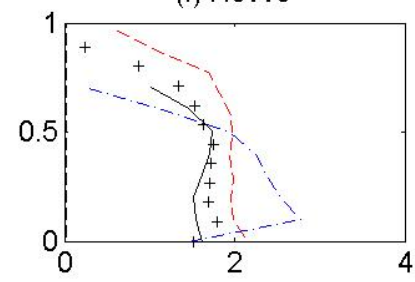

Figure 9: Horizontally averaged mean horizontal velocity, denoted by $U(z)$, as a function of normalized height $(z / h)$ using the raw PIV series. Every panel presents $\bar{U}(z)$ for all five scenarios - namely no vegetation (black + symbols, NV), rigid sparse (red dashed line, RS), rigid dense (blue dash and dotted line, RD), flexible sparse (thick green dots, FS) and flexible dense (black line, FD). Panels (a), (b) and (c) present the deep (H1), intermediate (H2) and shallow (H3) cases respectively for the slowest $U_{a}$ case (W1). Panels (d), (e) and (f) present the deep (H1), intermediate $(\mathrm{H} 2)$ and shallow $(\mathrm{H} 3)$ cases respectively for the intermediate $U_{a}$ case (W2). Similarly, panels (g), (h) and (i) present the deep (H1), intermediate (H2) and shallow (H3) cases respectively for the fastest $U_{a}$ case (W3). 

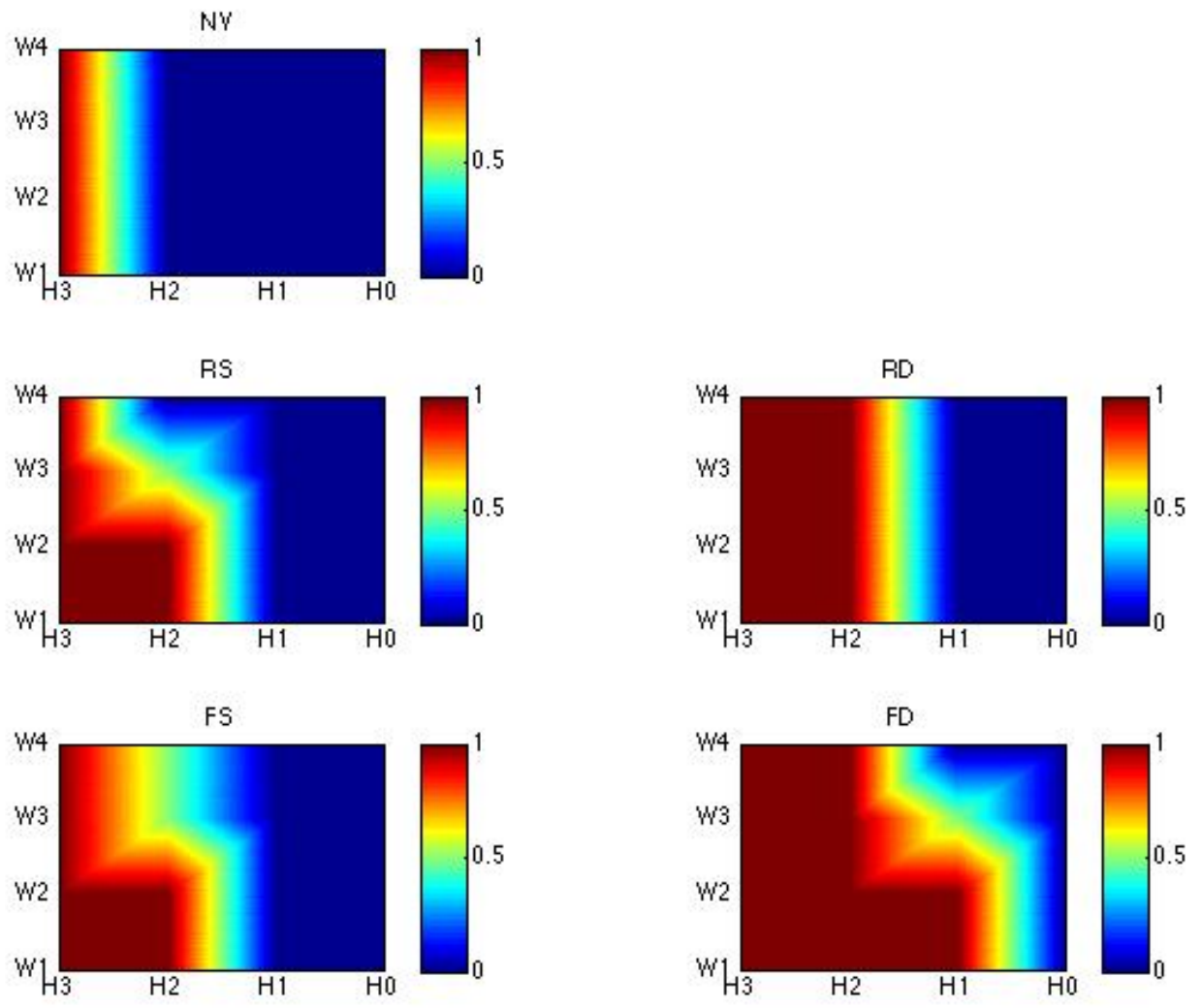

Figure 10: Key observations regarding $\bar{U}(z)$ for all the experiments in the form of a binary phase diagram. Panel (a) summarizes the results for the no vegetation (NV) scenario, panel (b) for rigid sparse (RS), panel (c) for rigid dense (RD), panel (d) for flexible sparse (FS) and panel (e) for flexible dense (FD) scenario. A value of 1 in the colormap indicates fully forward flow in the direction of $U_{a}$ and a value of 0 indicates existence of a counter-current flow. The transition from one type to flow to another is represented by a number in between 0 and 1 . 

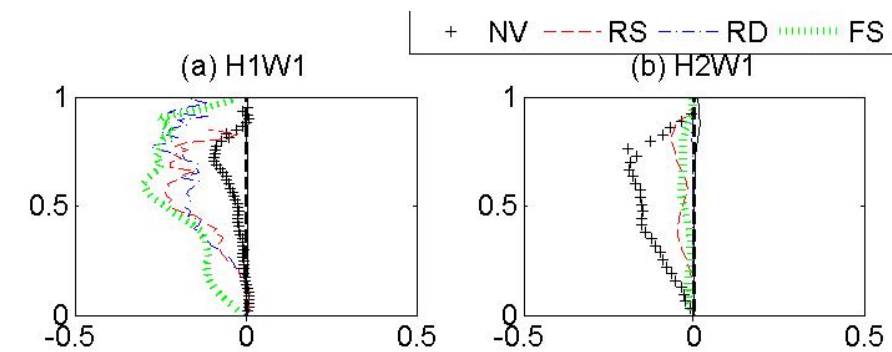

FS - FD

(c) H3W1
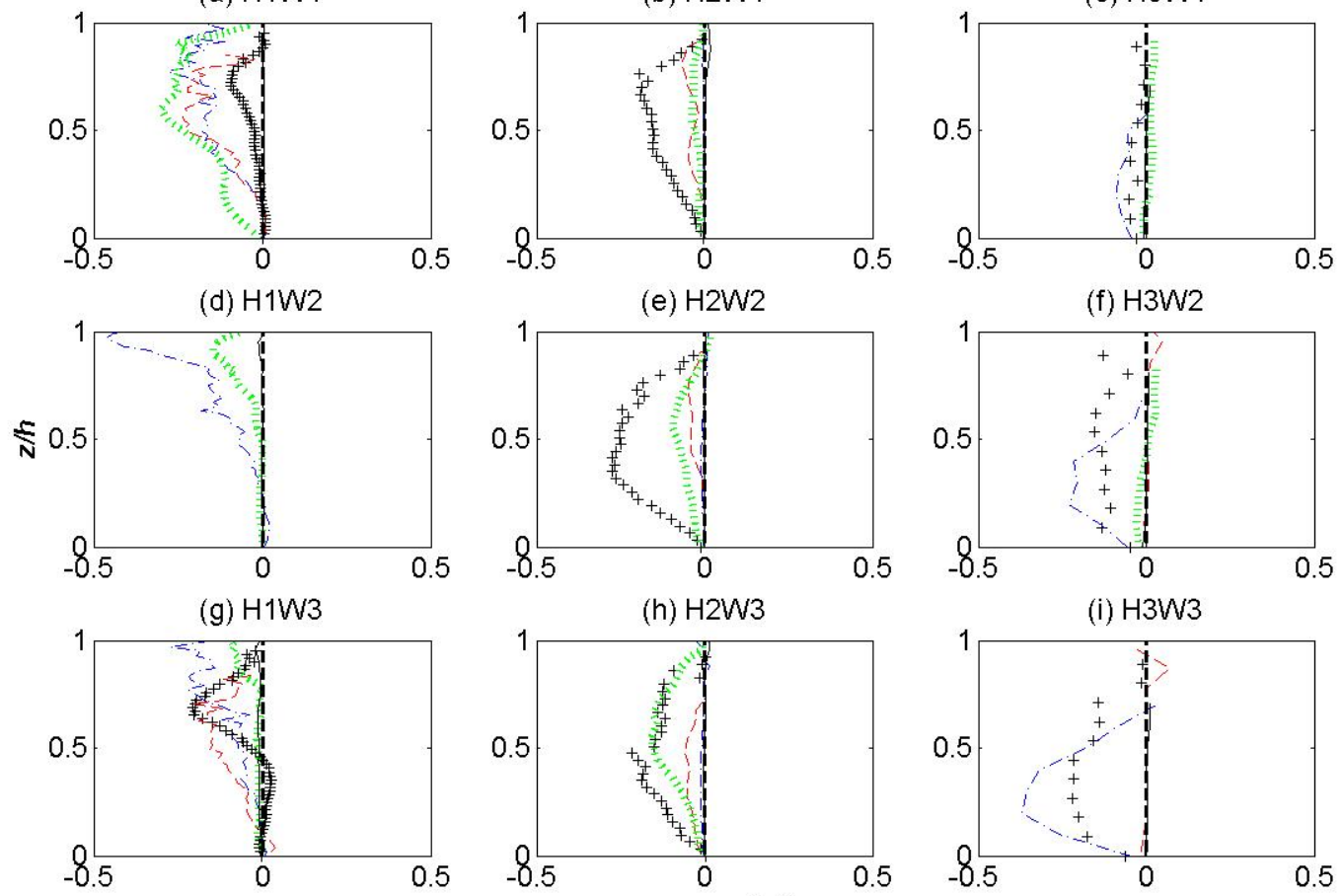

(h) $\mathrm{H}_{2} \mathrm{~W} 3$
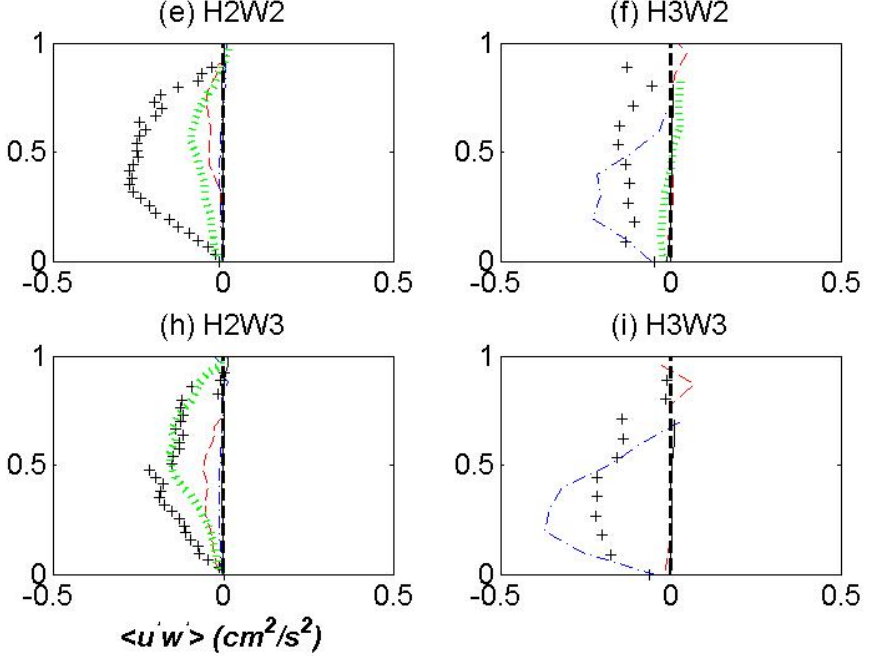

Figure 11: Same as Figure 9 but for the total stress $\left(\overline{u^{\prime} w^{\prime}}\right)$ computed from the raw PIV series (i.e. including noise, wave, and turbulence). 


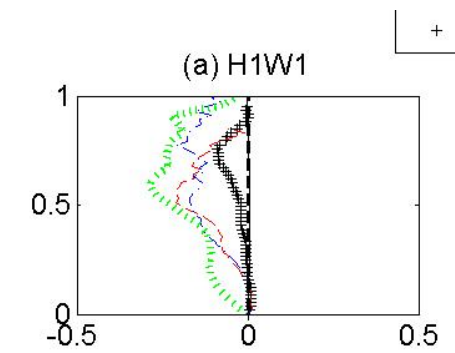

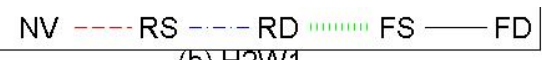
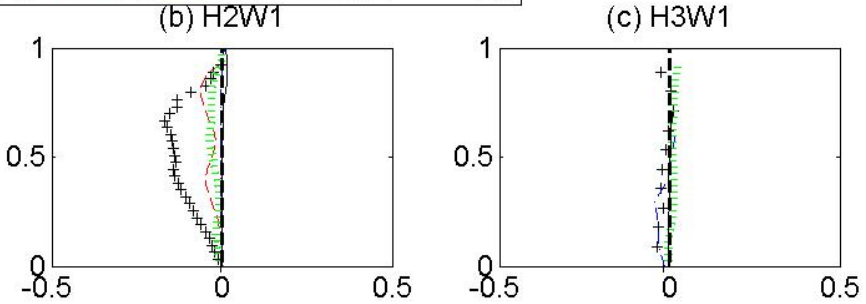

(d) $\mathrm{H} 1 \mathrm{~W} 2$

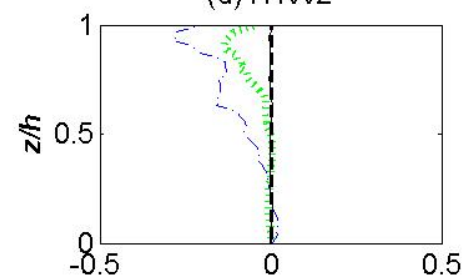

(e) $\mathrm{H} 2 \mathrm{~W} 2$
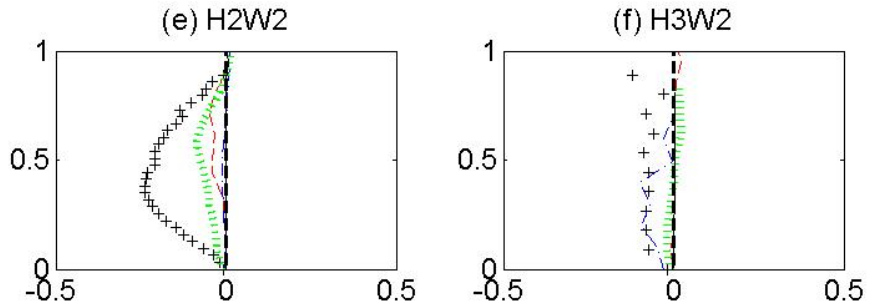

(g) $\mathrm{H} 1 \mathrm{~W} 3$

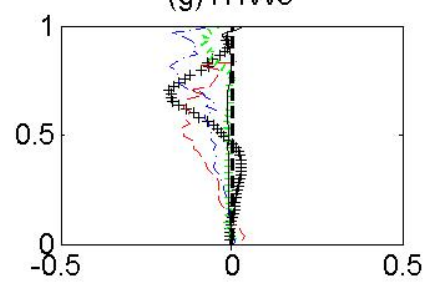

(h) $\mathrm{H}_{2} \mathrm{~W} 3$

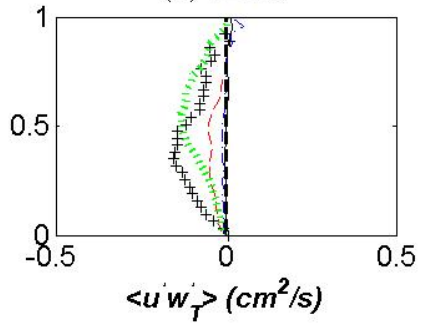

(i) $\mathrm{H}_{3} \mathrm{~W} 3$

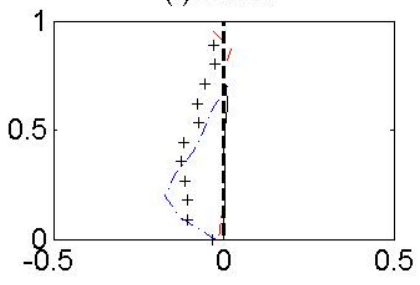

Figure 12: Same as Figure 9 but for the turbulent Reynolds stress $\left(\overline{u^{\prime} w^{\prime}}\right)$ computed from the separated turbulent series.

\section{Appendix A. Lorenz curve method}

Details on the Lorenz curve filtering method is briefly reviewed. As discussed in section 3.2 , it is necessary to find the inflection of the Lorenz curve. The following approach can be used to construct the Lorenz curve in the Fourier domain, to identify the inflection point and to separate the signal and noise in the frequency domain for a sample time series $u(t)$. The Fast Fourier Transform (FFT) of a velocity time series (say $u(t)$ ) is first computed (labeled as $F_{u(t)}$ ) and the energy content of $F_{u(t)}$, labeled $E_{F}$, is determined from the squared Fourier amplitudes (i.e. $\left.E_{F}=\operatorname{Real}\left(F_{u(t)}\right)^{2}+\operatorname{Imaginary}\left(F_{u(t)}\right)^{2}\right)$. These $E_{F}$ are sorted in ascending order from minimum to maximum energetic coefficient and their corresponding indices are tracked. The Lorenz curve is constructed by summing sequentially the coefficients and their associated $E_{F}$ normalized so that when all the Fourier coefficients are accounted for - the abscissa is unity, and this final point includes all the energy (or variance) in the original time so that - the ordinate is unity. Hence, when all the Fourier coefficients are eliminated, the energy content is zero and when all the Fourier coefficients are present, the entire energy or variance of the original series is accounted. By sequentially removing the most energetic coefficient and repeating the process until the least energetic coefficient is removed (on the abscissa) and plotting the concomitant drop in energy (on the ordinate), the Lorenz curve is obtained. The Lorenz curve is a convex curve describing 

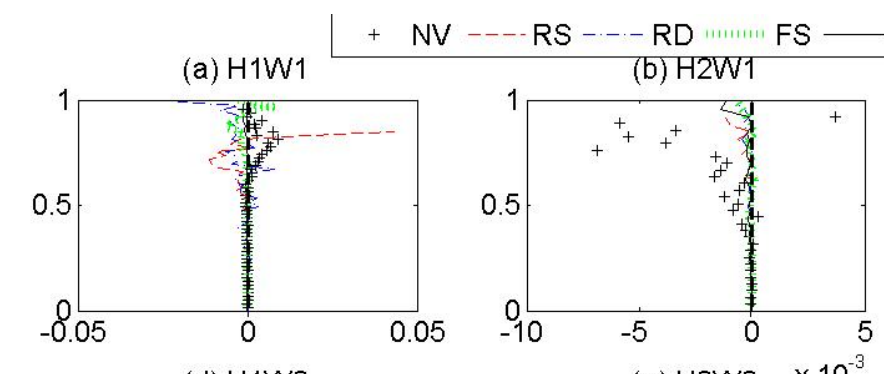

FD $\quad$ (c) H3W1

(d) H1W2

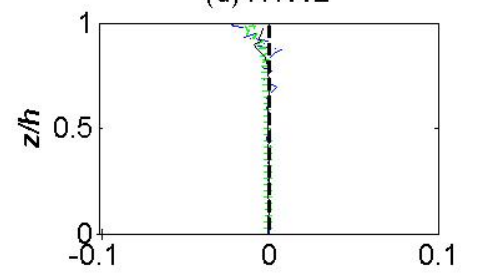

(e) H2W2 $\times 10^{-3}$
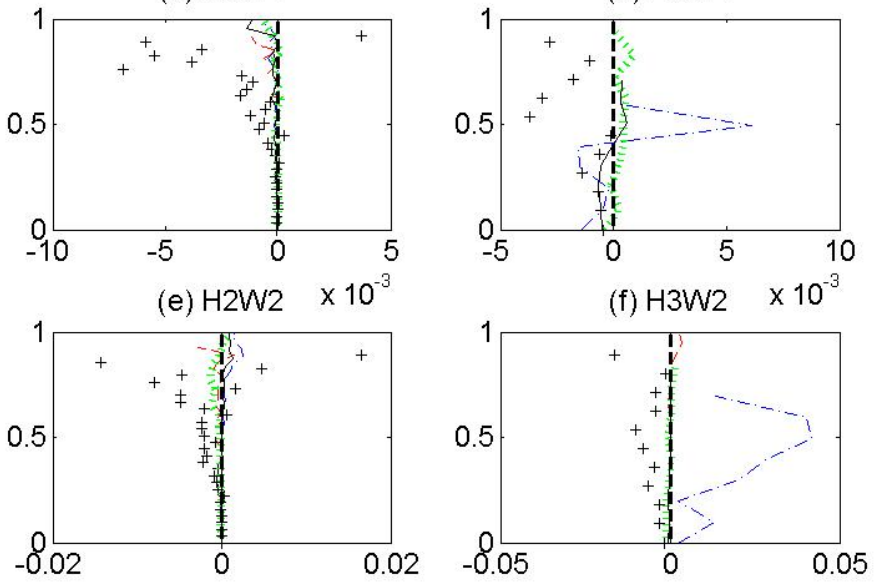

(g) $\mathrm{H}_{1} \mathrm{~W} 3$
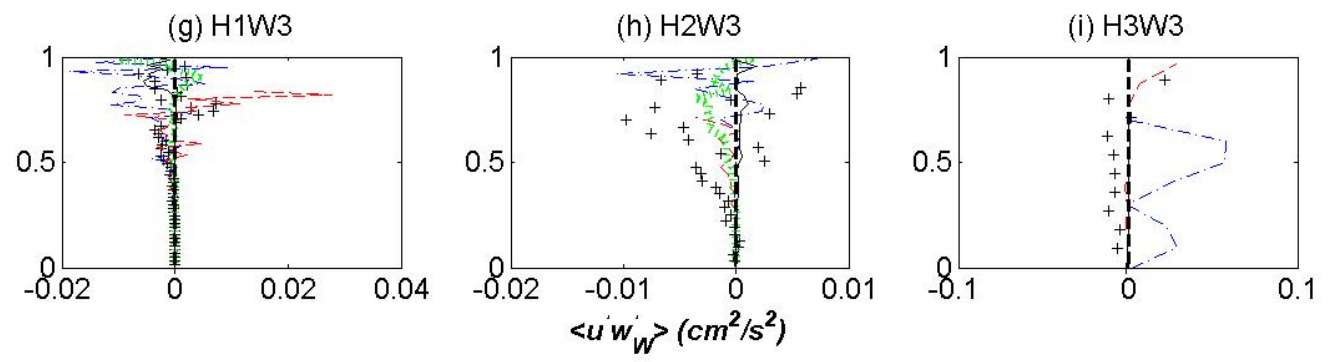

Figure 13: Same as Figure 9 but for the wave stress $\left(\overline{\overline{u^{\prime} w^{\prime}}}\right)$ computed from the separated wave series. Note here that the range of stress values are not identical across panels. 

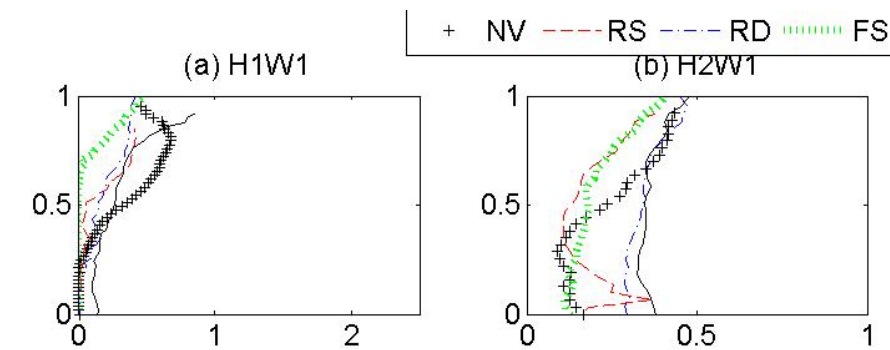

FS - FD
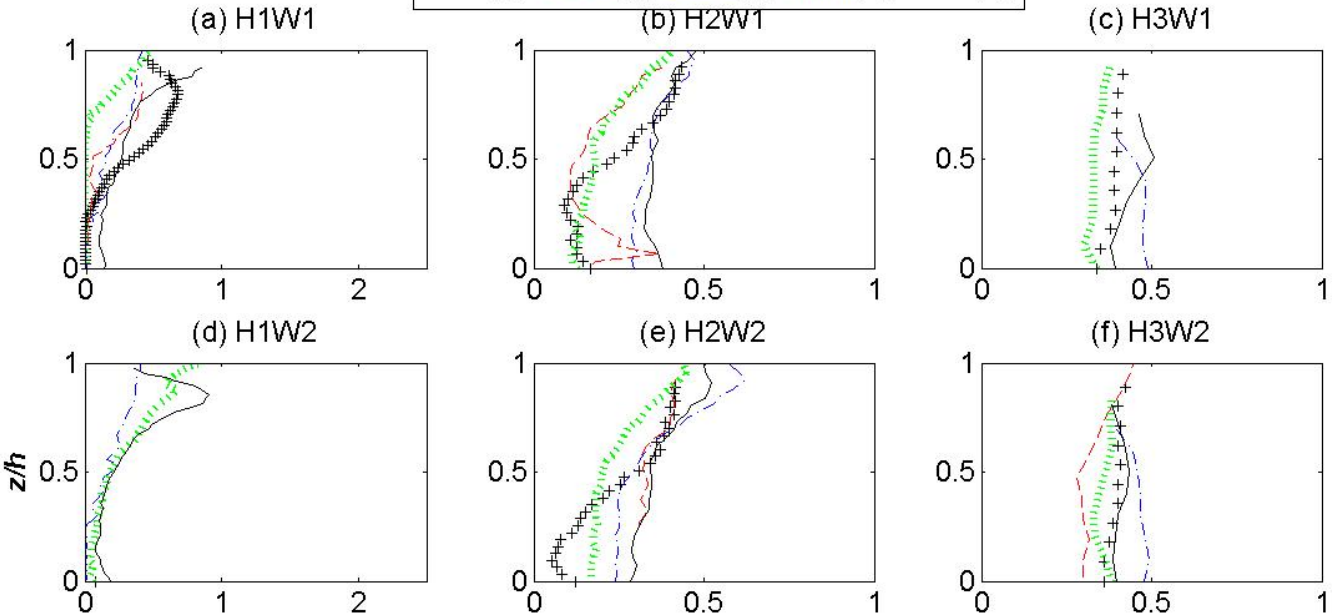

(e) $\mathrm{H} 2 \mathrm{~W} 2$

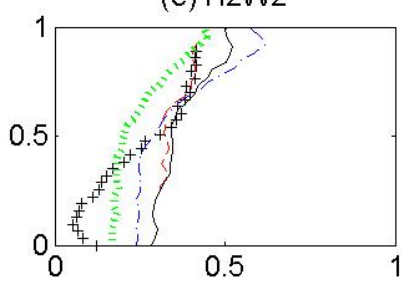

(f) $\mathrm{H} 3 \mathrm{~W} 2$

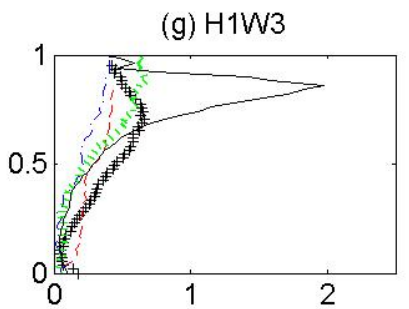

(h) $\mathrm{H}_{2} \mathrm{~W}_{3}$
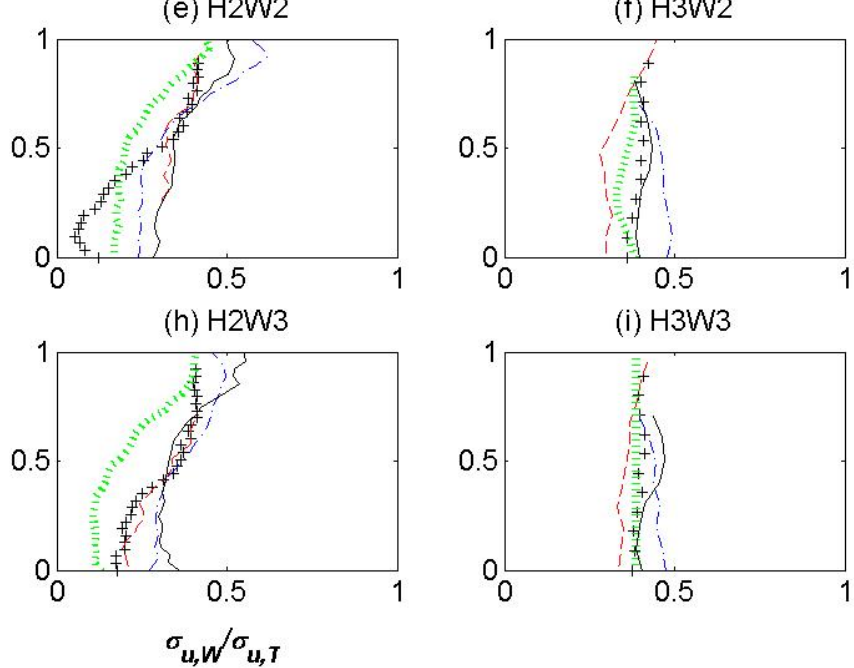

(i) $\mathrm{H} 3 \mathrm{~W} 3$

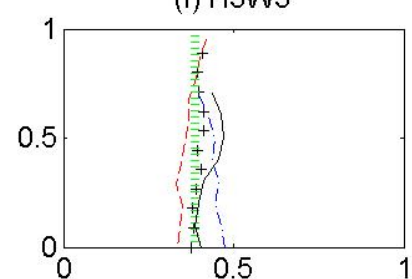

Figure 14: Same as Figure 9 but $\sigma_{u, W} / \sigma_{u, T}$. 

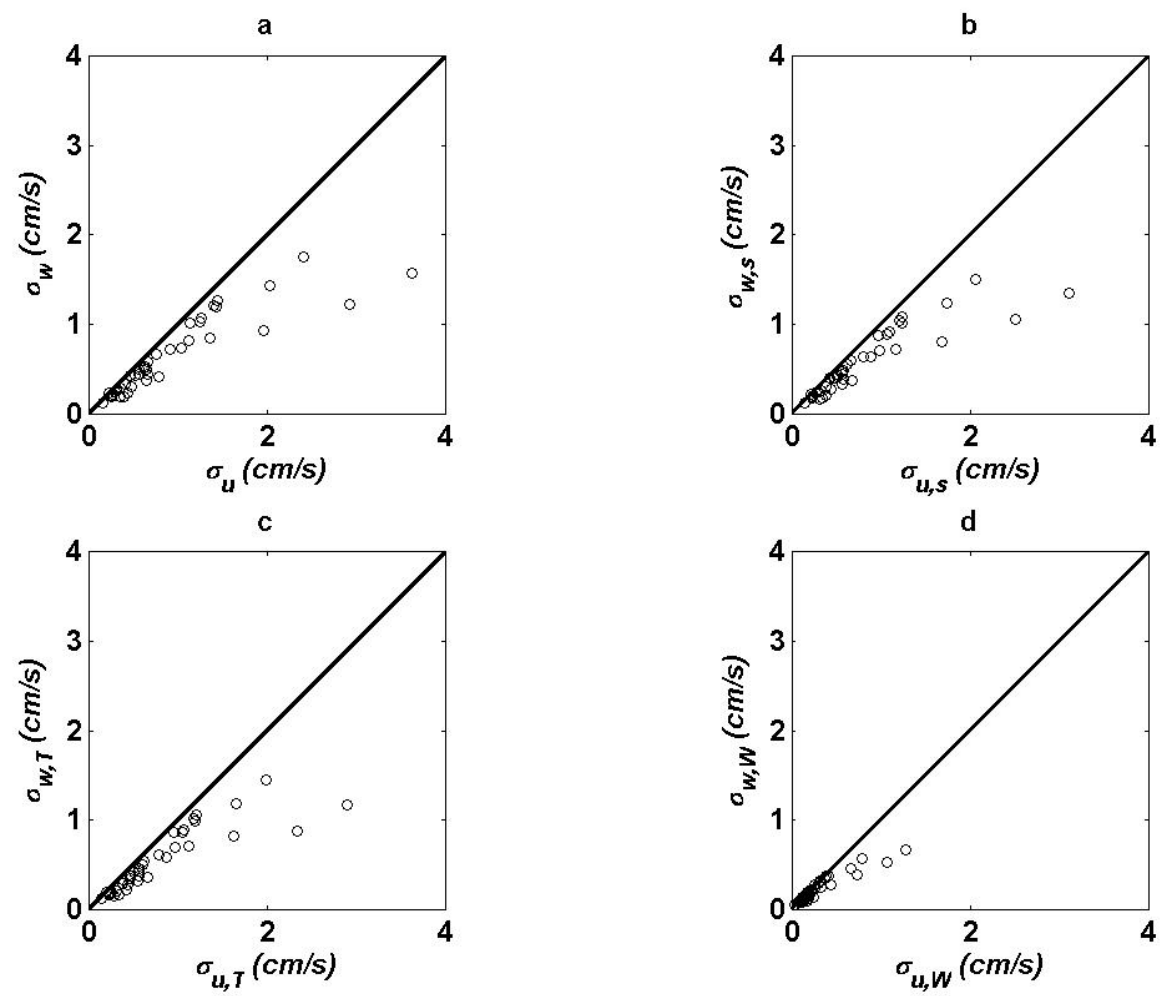

Figure 15: Comparison between the rms components of $u\left(=\sigma_{u}\right)$ and $w\left(=\sigma_{w}\right)$. Moreover, panel (a) uses the raw velocity obtained from PIV analysis, panel (b) for the series after filtering out the noise $\sigma_{u, s}$ and $\sigma_{w, s}$, panel (c) uses the separated turbulent series $\sigma_{u, T}$ and $\sigma_{w, T}$ and panel (d) uses the separated wave series $\sigma_{u, W}$ and $\sigma_{w, W}$. The one-to-one line is also shown for reference. 
the cumulative energy (ordinate, [0-1]) contained in the smallest energy components (abscissa, [0-1]). A sample Lorenz curve is shown in Figure A.16 for the $u(t)$ for the flexible dense scenario for H1W3 (deepest flow, fastest wind) configuration. The convexity of the curve relative to the diagonal or one-to-one line (corresponding to a perfectly balanced energy distribution) is directly proportional to the energy disbalance shown in Figure A.16. Hence, based on the convexity of the Lorenz curve, a global thresholding criterion can be formulated whose premise is as follows: an energy cutoff can be selected such that the proportion at which the gain (in parsimony) by hard thresholding (or setting to zero its energy content) an additional $E_{F}$ coefficient is smaller than the loss in energy. This point on the Lorenz curve corresponds to the maximum convexity shown in Figure A.16. The normalization of the abscissa and ordinate of the Lorenz curve is needed so that fraction of coefficients and energy losses are measured on a scale of $0-1$ and are equally weighted. After the coefficients associated with the 'signal' (i.e. the more energetic ones) and the noise 'the least energetic ones' are delineated in terms of their position on the Lorenz curve, the Inverse Fast Fourier Transform (IFFT) of the thresholded and non-thresholded coefficients can be computed to separately construct the noise and signal time series, respectively, assuming phase-angles are not altered. This is the outcome of the series in Figure 5.

\section{Appendix B. Frequency filtering to separate wave and turbulence}

After the signal is isolated in the frequency domain $\left(F_{S}\right)$, the following algorithm is added to separate the wave and turbulence from the signal. A frequency window $(2-5 \mathrm{~Hz})$ is chosen, the extremities of which are labeled as $f_{l}$ (low frequency bound) and $f_{h}$ (high frequency bound). The amplitude $\left(A_{S}\right)$ (obtained by taking square root of the sum of the squares of the real and imaginary parts) and phase information $(\phi)$ of the signal within the frequency range are determined. Fit a straight line between the amplitudes at the extremities between $f_{l}$ and $f_{h}$. The interpolated straight line constitutes the amplitude of the turbulence signal $\left(A_{t}\right)$. Compute the amplitude of the wave from $A_{w}^{2}=A_{S}^{2}-A_{t}^{2}$. Determine the Fourier coefficients of the wave by $F_{w}=$ $A_{w}[\cos (\phi)+i \sin (\phi)]$ and turbulence by $F_{t}=A_{t}[\cos (\phi)+i \sin (\phi)]$. Compute IFFT on $F_{w}$ and $F_{t}$ to reconstruct the wave and turbulent time series. 


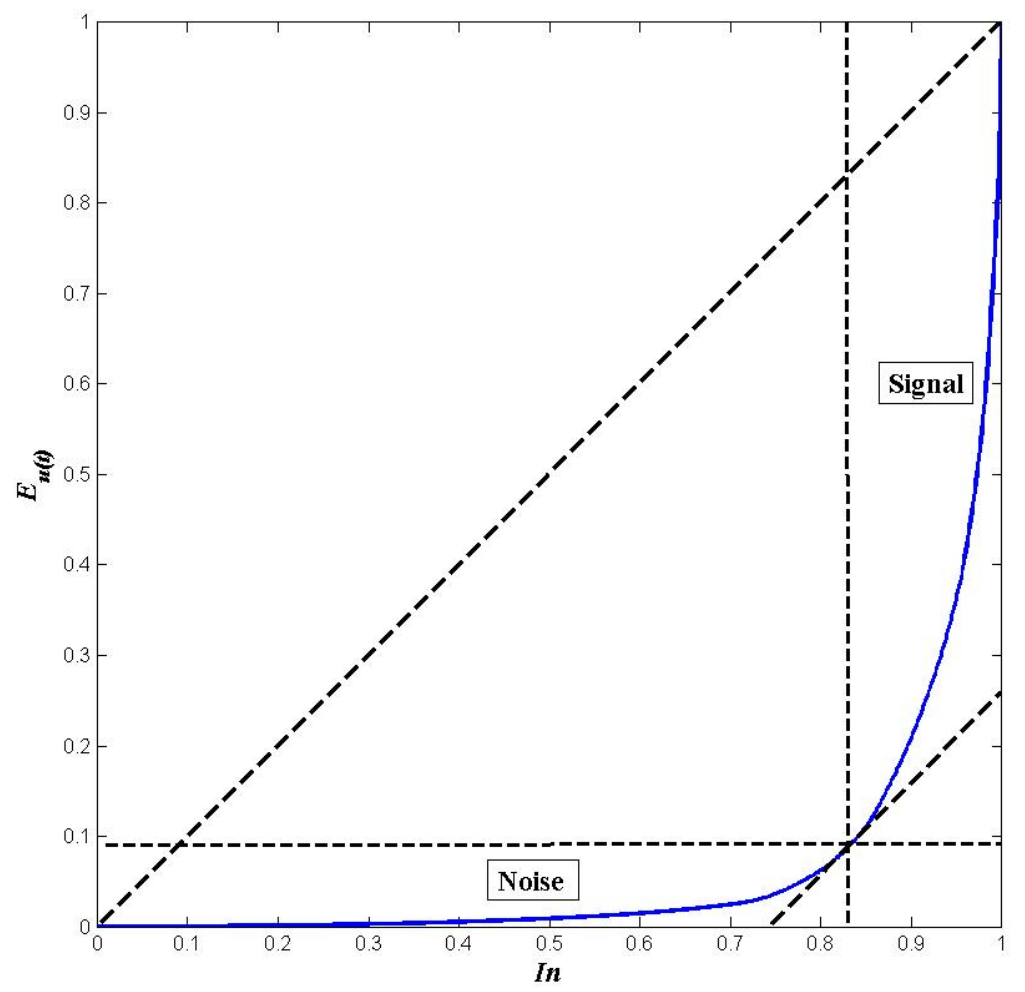

Figure A.16: Lorenz curve (blue line) constructed following the procedure described in appendix Appendix A. The abscissa indicates the fraction of Fourier coefficients (In) associated with the original time series (unity indicates all Fourier coefficients are present) and the ordinate indicates the cumulative energy or squared Fourier amplitudes (unity indicates all the variance in the original series is explained when all Fourier coefficients are included). The dashed lines indicate the point computed from the maximum inflection point coinciding with the slope of the one-to-one line. 
M. Begon, J. Harper, C. Towsend. 1999. Ecology: individuals, populations and communities, Blackwell Science, London, 1986.

S. J. Bennett, T. Pirim, B. D. Barkdoll, Using simulated emergent vegetation to alter stream flow direction within a straight experimental channel, Geomorphology 44 (1) (2002) 115-126, ISSN 0169-555X.

R. Costanza, R. d'Arge, R. De Groot, S. Farber, M. Grasso, B. Hannon, K. Limburg, S. Naeem, R. V. O’ Neill, J. Paruelo, et al., The value of the world's ecosystem services and natural capital, Ecological Economics 25 (1) (1998) 3-15.

M. Fonseca, J. S. Fisher, A comparison of canopy friction and sediment movement between four species of seagrass with reference to their ecology and restoration, Marine Ecology Progress Series 29 (1986) 15-22.

M. S. Fonseca, J. A. Cahalan, A preliminary evaluation of wave attenuation by four species of seagrass, Estuarine, Coastal and Shelf Science 35 (6) (1992) 565-576.

M. C. Gambi, A. R. Nowell, P. Jumars, Flume observations on flow dynamics in Zostera marina(eelgrass) beds., Marine ecology progress series. Oldendorf 61 (1) (1990) 159-169.

M. L. Gleason, D. A. Elmer, N. C. Pien, J. S. Fisher, Effects of stem density upon sediment retention by salt marsh cord grass, Spartina alterniflora Loisel, Estuaries 2 (4) (1979) 271-273.

A. A. Jordanova, C. James, Experimental study of bed load transport through emergent vegetation, Journal of Hydraulic Engineering 129 (6) (2003) 474-478, ISSN 0733-9429.

H. M. Nepf, Drag, turbulence, and diffusion in flow through emergent vegetation, Water Resources Research 35 (2) (1999) 479-489, ISSN 0043-1397.

H. M. Nepf, Flow and transport in regions with aquatic vegetation, Annual Reviews of Fluid Mechanics 44 (2012a) 123-142.

C. Pergent-Martini, V. Pasqualini, L. Ferrat, G. Pergent, Ecological data in integrated coastal zone management: case study of Posidonia oceanica meadows along the Corsican coastline (Mediterranean Sea), Environmental Management 38 (6) (2006) 889-895.

C. Prigent, E. Matthews, F. Aires, W. B. Rossow, Remote sensing of global wetland dynamics with multiple satellite data sets, Geophysical Research Letters 28 (24) (2001) 4631-4634.

T. B. Reusch, A. R. Chapman, Storm effects on eelgrass ( Zostera marina) and blue mussel ( Mytilus edulis) beds, Journal of Experimental Marine Biology and Ecology 192 (2) (1995) 257-271.

L. G. Ward, W. Michael Kemp, W. R. Boynton, The influence of waves and seagrass communities on suspended particulates in an estuarine embayment, Marine Geology 59 (1) (1984) 85-103.

G. G. Katul, D. Poggi, L. Ridolfi, A flow resistance model for assessing the impact of vegetation on flood routing mechanics, Water Resources Research 47 (8) (2011) W08533.

J. D. Fairbanks, P. Diplas, Turbulence characteristics of flows through partially and fully submerged vegetation, in: Proc., Wetlands Engineering and River Restoration Conf.: Engineering Approaches to Ecosystem Restoration, 865-870, 1998.

E. Kubrak, J. Kubrak, P. M. Rowieski, Vertical velocity distributions through and above submerged, flexible vegetation, Hydrological Sciences Journal 53 (4) (2008) 905-920, ISSN 0262-6667.

J. K. Lee, L. C. Roig, H. L. Jenter, H. M. Visser, Drag coefficients for modeling flow through emergent vegetation in the Florida Everglades, Ecological Engineering 22 (4-5) (2004) 237-248, ISSN 0925-8574.

A. Montakhab, B. Yusuf, A. Ghazali, T. Mohamed, Flow and sediment transport in vegetated waterways: a review, Reviews in Environmental Science and Bio/Technology 11 (3) (2012) 275-287, ISSN 1569-1705.

H. M. Nepf, E. W. Koch, Vertical secondary flows in submersed plant-like arrays, Limnology and Oceanography 44 (4) (1999) 1072-1080, ISSN 0024-3590.

H. M. Nepf, E. R. Vivoni, Flow structure in depth-limited, vegetated flow, Journal of Geophysical Research-Oceans 105 (C12) (2000) 28547-28557, ISSN 0148-0227.

M. Righetti, Flow analysis in a channel with flexible vegetation using double-averaging method, Acta Geophysica 56 (3) (2008) 801-823, ISSN 1895-6572.

A. N. Sukhodolov, T. A. Sukhodolova, Case study: Effect of submerged aquatic plants on turbulence structure in a lowland river, Journal of Hydraulic Engineering 136 (7) (2009) 434-446, ISSN 0733-9429.

X. Sun, K. Shiono, Flow resistance of one-line emergent vegetation along the floodplain edge of a compound open channel, Advances in Water Resources 32 (3) (2009) 430-438, ISSN 0309-1708.

Y. Tanino, H. M. Nepf, Laboratory investigation of mean drag in a random array of rigid, emergent cylinders, Journal of Hydraulic Engineering 134 (1) (2008) 34-41, ISSN 0733-9429.

C. Wilson, T. Stoesser, P. Bates, A. B. Pinzen, Open channel flow through different forms of submerged flexible vegetation, Journal of Hydraulic Engineering 129 (11) (2003) 847-853, ISSN 0733-9429.

Y. H. Huang, J. E. Saiers, J. W. Harvey, G. B. Noe, S. Mylon, Advection, dispersion, and filtration of fine particles within emergent vegetation of the Florida Everglades, Water Resources Research 44(4) (2008) W04408, ISSN 0043-1397.

A. F. Lightbody, H. M. Nepf, Prediction of velocity profiles and longitudinal dispersion in emergent salt marsh vegetation, Limnology and Oceanography 51 (1) (2006) 218-228, ISSN 0024-3590.

E. Murphy, M. Ghisalberti, H. Nepf, Model and laboratory study of dispersion in flows with submerged vegetation, Water 
Resources Research 43 (5) (2007) W05438, ISSN 0043-1397.

H. M. Nepf, J. A. Sullivan, R. A. Zavistoski, A model for diffusion within emergent vegetation, Limnology and Oceanography 42 (8) (1997a) 1735-1745, ISSN 0024-3590.

H. M. Nepf, C. G. Mugnier, R. A. Zavistoski, The effects of vegetation on longitudinal dispersion, Estuarine Coastal and Shelf Science 44 (6) (1997b) 675-684, ISSN 0272-7714.

T. Serra, H. J. S. Fernando, R. V. Rodriguez, Effects of emergent vegetation on lateral diffusion in wetlands, Water Research 38 (1) (2004) 139-147, ISSN 0043-1354.

D. Poggi, A. Porporato, L. Ridolfi, J. Albertson, G. Katul, The effect of vegetation density on canopy sub-layer turbulence, Boundary-Layer Meteorology 111 (3) (2004) 565-587.

M. Raupach, A. Thom, Turbulence in and above Plant canopies, Annual Reviews of Fluid Mechanics 13 (1981) 97-129.

J. Finnigan, Turbulence in plant canopies, Annual Reviews of Fluid Mechanics 32 (2000) 519-571.

M. Baptist, A flume experiment on sediment transport with flexible, submerged vegetation, in: International Workshop on Riparian Forest Vegetated Channels: Hydraulic, Morphological and Ecological Aspects, RIPFOR, Trento, Italy, 2003.

A. A. Jordanova, C. S. James, A. L. Birkhead, Practical estimation of flow resistance through emergent vegetation, Proceedings of the Institution of Civil Engineers-Water Management 159 (3) (2006) 173-181, ISSN 1741-7589.

C. Liu, Y.-m. Shen, Flow structure and sediment transport with impacts of aquatic vegetation, Journal of Hydrodynamics, Ser. B 20 (4) (2008) 461-468, ISSN 1001-6058.

U. Neumeier, P. Ciavola, Flow resistance and associated sedimentary processes in a Spartina maritima salt-marsh, Journal of Coastal Research 20 (2) (2004) 435-447, ISSN 1551-5036.

B. H. Schmid, U. Stephan, M. A. Hengl, Sediment deposition in constructed wetland ponds with emergent vegetation: laboratory study and mathematical model, Water Science and Technology 51 (9) (2005) 307-314, ISSN 0273-1223.

R. G. Sharpe, C. S. James, Deposition of sediment from suspension in emergent vegetation, Water Sa 32 (2) (2006) 211-218, ISSN 0378-4738.

J. Shucksmith, J. Boxall, I. Guymer, Bulk flow resistance in vegetated channels: Analysis of momentum balance approaches based on data obtained in aging live vegetation, Journal of Hydraulic Engineering 137 (12) (2011) 16241635, ISSN 0733-9429.

U. Stephan, M. Hengl, B. H. Schmid, Sediment retention in constructed wetland ponds - A laboratory study, Journal of Environmental Science and Health, Part a-Toxic/Hazardous Substances \& Environmental Engineering 40 (6-7) (2005) 1415-1430, ISSN 1093-4529.

L. J. Zong, H. Nepf, Spatial distribution of deposition within a patch of vegetation, Water Resources Research 47 (2011) W03516, ISSN 0043-1397.

H. Charnock, Wind stress on a water surface, Quarterly Journal of the Royal Meteorological Society (1955) 639-640.

G. Csanady, Air-Sea Interactions: Laws and Mechanisms, Cambridge University Press, Cambridge, England, 2004.

B. Hellström, Wind effect on lakes and rivers, Generalstabens litografiska anstalts förlag, 1941.

I. K. Tsanis, Simulation of wind-induced water currents, Journal of Hydraulic Engineering 115 (8) (1989) 1113-1134.

W. D. Baines, D. Knapp, Wind driven water currents, Journal of Hydraulic Divisions, ASCE 91 (1965) 205-221.

T. K. Cheung, R. L. Street, The turbulent layer in the water at an airwater interface, Journal of Fluid Mechanics 194 (1988) 133-151.

L. H. Goossens, H. J. Van Pagee, P. J. Tessel, Vertical turbulent diffusion in air-driven water flows, Journal of the Hydraulics Division 108 (9) (1982) 995-1009.

S. Komori, R. Nagaosa, Y. Murakami, Turbulence structure and mass transfer across a sheared air-water interface in wind-driven turbulence, Journal of Fluid Mechanics 249 (1993) 161-183.

C. Kranenburg, Turbulent surface boundary-layer induced by an off-shore wind, Journal of Hydraulic Research 25 (1) (1987) 53-65.

I. Langmuir, et al., Surface motion of water induced by wind, Science 87 (2250) (1938) 119-123.

S. Longo, L. Chiapponi, M. Clavero, T. Mäkelä, D. Liang, Study of the turbulence in the air-side and water-side boundary layers in experimental laboratory wind induced surface waves, Coastal Engineering 69 (2012) 67-81.

M. Sanjou, I. Nezu, A. Toda, PIV studies on turbulence structure in air/water interface with wind-induced water waves, Journal of Hydrodynamics, Ser. B 22 (5) (2010) 349-353.

M. Sanjou, I. Nezu, Turbulence structure and coherent vortices in open-channel flows with wind-induced water waves, Environmental Fluid Mechanics 11 (2) (2011) 113-131.

I. K. Tsanis, H. J. Leutheusser, The structure of turbulent shear-induced countercurrent flow, Journal of Fluid Mechanics 189 (1988) 531-552.

J. Wu, Wind-induced drift currents, Journal of Fluid Mechanics 68 (01) (1975) 49-70.

A. Bukatov, D. Zavyalov, Wind-induced motion of water in shallow-water closed basins, Physical Oceanography 14 (5) (2004) 284-294.

S. Belcher, J. Harris, R. Street, Linear dynamics of wind waves in coupled turbulent air water flow, Part 1. Theory, Journal of Fluid Mechanics 271 (1) (1994) 119-151, ISSN 1469-7645. 
F. Cioffi, F. Gallerano, E. Napoli, Three-dimensional numerical simulation of wind-driven flows in closed channels and basins, Journal of Hydraulic Research 43 (3) (2005) 290-301.

J. Harris, S. Belcher, R. Street, Linear dynamics of wind waves in coupled turbulent air"water flow. Part 2. Numerical model, Journal of Fluid Mechanics 308 (1) (1996) 219-254, ISSN 1469-7645.

J. Hunter, C. Hearn, Lateral and vertical variations in the wind-driven circulation in long, shallow lakes, Journal of Geophysical Research: Oceans (1978-2012) 92 (C12) (1987) 13106-13114.

X. Jin, C. Kranenburg, Quasi-3D numerical modeling of shallow-water circulation, Journal of Hydraulic Engineering 119 (4) (1993) 458-472.

M. B. Koçyigit, Ö. Koçyigit, Numerical study of wind-induced currents in enclosed homogeneous water bodies., Turkish Journal of Engineering \& Environmental Sciences 28 (3) (2004) 207-21.

Z. Meyer, Wind set-up of water level in a river, Acta Geophysica 59 (2) (2011) 317-333.

W. Rodi, Turbulent models and their application in hydraulicsa state of the art review, International Association for Hydraulics Research, Delft, 1980.

J. Wu, I. K. Tsanis, Numerical study of wind-induced water currents, Journal of Hydraulic Engineering 121 (5) (1995) 388-395.

Y. Yang, Wind induced countercurrent flow in shallow water, Ph.D. thesis, University of Western Ontario, London, Ontario, Canada, 2001.

Y. Yang, A. Straatman, H. Hangan, E. Yanful, An engineering model for countercurrent flow under wind-induced waves and current, Environmental Fluid Mechanics 8 (1) (2008) 19-29.

K. Bradley, C. Houser, Relative velocity of seagrass blades: Implications for wave attenuation in low-energy environments, Journal of Geophysical Research: Earth Surface (2003-2012) 114 (F1) (2009) F01004.

J. C. Hansen, M. A. Reidenbach, Wave and tidally driven flows in eelgrass beds and their effect on sediment suspension, Marine Ecology Progress Series 448 (2011) 271-287.

E. Infantes, A. Orfila, G. Simarro, J. Terrados, M. Luhar, H. Nepf, Effect of a seagrass (Posidonia oceanica) meadow on wave propagation, Marine Ecology Progress Series 456 (2012) 63-72, ISSN 0171-8630.

N. Kobayashi, A. W. Raichle, T. Asano, Wave attenuation by vegetation, Journal of Waterway, Port, Coastal, and Ocean Engineering 119 (1) (1993) 30-48.

E. Manca, I. Caceres, J. Alsina, V. Stratigaki, I. Townend, C. Amos, Wave energy and wave-induced flow reduction by full-scale model Posidonia oceanica seagrass, Continental Shelf Research 50 (2012) 100-116.

F. J. Mendez, I. J. Losada, An empirical model to estimate the propagation of random breaking and nonbreaking waves over vegetation fields, Coastal Engineering 51 (2) (2004) 103-118.

K. C. Riffe, S. M. Henderson, J. C. Mullarney, Wave dissipation by flexible vegetation, Geophysical Research Letters 38 (18) (2011) L18607.

F. I. Thomas, C. D. Cornelisen, Ammonium uptake by seagrass communities: Effects of oscillatory versus unidirectional flow, Marine Ecology Progress Series 247 (1) (2003) 51-57.

H. Coops, G. Van der Velde, Effects of waves on helophyte stands: mechanical characteristics of stems of Phragmites australis and Scirpus lacustris, Aquatic Botany 53 (3) (1996) 175-185.

M. Luhar, S. Coutu, E. Infantes, S. Fox, H. Nepf, Wave-induced velocities inside a model seagrass bed, Journal of Geophysical Research-Oceans 115(12) (2010) C12005, ISSN 0148-0227.

R. J. Lowe, J. R. Koseff, S. G. Monismith, Oscillatory flow through submerged canopies: 1. Velocity structure, Journal of Geophysical Research: Oceans (1978-2012) 110 (C10) (2005a) C10016.

M. A. Reidenbach, J. R. Koseff, S. G. Monismith, Laboratory experiments of fine-scale mixing and mass transport within a coral canopy, Physics of Fluids (1994-present) 19 (7) (2007) 075107.

Y. Tanino, H. M. Nepf, P. S. Kulis, Gravity currents in aquatic canopies, Water Resources Research 41 (12) (2005) W12402, ISSN 0043-1397.

C. Li, K. Yan, Numerical investigation of wave current vegetation interaction, Journal of Hydraulic Engineering 133 (7) (2007) 794-803, ISSN 0733-9429.

C. Li, M. Zhang, 3D modelling of hydrodynamics and mixing in a vegetation field under waves, Computers \& Fluids 39 (4) (2010) 604-614, ISSN 0045-7930.

J. C. Mullarney, S. M. Henderson, Wave-forced motion of submerged single-stem vegetation, Journal of Geophysical Research-Oceans 115 (2010) C12061, ISSN 0148-0227.

E. W. Koch, G. Gust, Water flow in tide-and wave-dominated beds of the seagrass Thalassia testudinum, Marine Ecology Progress Series 184 (1) (1999) 63-72.

I. Huang, J. Rominger, H. Nepf, The motion of kelp blades and the surface renewal model, Limnology and Oceanography 56 (4) (2011) 1453-1462, ISSN 0024-3590.

R. J. Lowe, J. R. Koseff, S. G. Monismith, J. L. Falter, Oscillatory flow through submerged canopies: 2. Canopy mass transfer, Journal of Geophysical Research: Oceans (1978-2012) 110 (C10) (2005b) C10017.

H. M. Nepf, Hydrodynamics of vegetated channels, Journal of Hydraulic Research 50 (3) (2012b) 262-279, ISSN 00221686. 
M. Ghisalberti, H. M. Nepf, Mixing layers and coherent structures in vegetated aquatic flows, Journal of Geophysical Research: Oceans (1978-2012) 107 (C2) (2002) 3-1.

M. Ghisalberti, H. Nepf, The structure of the shear layer in flows over rigid and flexible canopies, Environmental Fluid Mechanics 6 (3) (2006) 277-301, ISSN 1567-7419.

M. Ghisalberti, H. Nepf, Shallow flows over a permeable medium: The hydrodynamics of submerged aquatic canopies, Transport in Porous Media 78 (3) (2009) 385-402, ISSN 0169-3913.

H. Nepf, M. Ghisalberti, Flow and transport in channels with submerged vegetation, Acta Geophysica 56 (3) (2008) 753-777, ISSN 1895-6572.

T.-a. Okamoto, I. Nezu, Spatial evolution of coherent motions in finite-length vegetation patch flow, Environmental Fluid Mechanics 13 (5) (2013) 417-434.

C. E. Oldham, J. J. Sturman, The effect of emergent vegetation on convective flushing in shallow wetlands: Scaling and experiments, Limnology and Oceanography 46 (6) (2001) 1486-1493, ISSN 0024-3590.

I. Nezu, M. Sanjou, Turburence structure and coherent motion in vegetated canopy open-channel flows, Journal of Hydro-environment Research 2 (2) (2008) 62-90.

W. Thielicke, E. J. Stamhuis, PIVlab-time-resolved digital particle image velocimetry tool for matlab, Published under the BSD license, programmed with MATLAB 7 (0.246) (2010) R14.

M. Raupach, R. Shaw, Averaging procedures for flow within vegetation canopies, Boundary-Layer Meteorology 61 (1982) 47-64.

M. O. Lorenz, Methods of measuring the concentration of wealth, Publications of the American Statistical Association 9 (70) (1905) 209-219.

G. G. Katul, B. Vidakovic, Identification of low-dimensional energy-containing/flux transporting eddy motion in the atmospheric surface layer using wavelet thresholding methods, Journal of the Atmospheric Sciences 55 (1998) 377389.

B. Vidakovic, Statistical modeling by wavelets, John Wiley \& Sons, 2009.

G. G. Katul, B. Vidakovic, The partitioning of attached and detached eddy motion in the atmospheric surface layer using Lorenz wavelet filtering, Boundary Layer Meteorology 77 (1996) 153-172.

T. Banerjee, G. Katul, Logarithmic scaling in the longitudinal velocity variance explained by a spectral budget, Physics of Fluids (1994-present) 25 (12) (2013) 125106.

B. Kader, A. Yaglom, Spectra and correlation functions of surface layer atmospheric turbulence in unstable thermal stratification, in: Turbulence and Coherent Structures, Springer, 387-412, 1991.

G. G. Katul, J. Albertson, C. Hsieh, P. Conklin, J. Sigmon, M. Parlange, K. Knoerr, The inactive eddy-motion and the large-scale turbulent pressure fluctuations in the dynamic sublayer, Journal of the Atmospheric Sciences 53 (1996) 2512-2524.

G. G. Katul, C. Chu, A theoretical and experimental investigation of the energy-containing scales in the dynamic sublayer of boundary-layer flows, Boundary Layer Meteorology 86 (1998) 279-312.

V. Nikora, Origin of the -1 spectral law in wall-bounded turbulence, Physical Review Letters 83 (4) (1999) 734.

A. Perry, M. Chong, On the mechanism of wall turbulence, Journal of Fluid Mechanics 119 (1982) 173-217. 